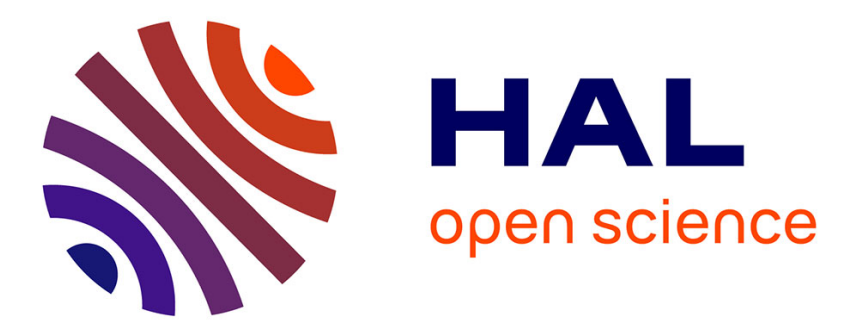

\title{
Supramolecular organization and biological interaction of the squalenoyl siRNA nanoparticles
}

\author{
Marie Caillaud, Frédéric Gobeaux, Miryana Hémadi, Suzan Boutary, Patrick \\ Guenoun, Didier Desmaële, Patrick Couvreur, Frank Wien, Fabienne Testard, \\ Liliane Massaad-Massade
}

\section{To cite this version:}

Marie Caillaud, Frédéric Gobeaux, Miryana Hémadi, Suzan Boutary, Patrick Guenoun, et al.. Supramolecular organization and biological interaction of the squalenoyl siRNA nanoparticles. International Journal of Pharmaceutics, 2021, 609, pp.121117. 10.1016/j.ijpharm.2021.121117 . hal03379142

\section{HAL Id: hal-03379142 \\ https://hal.science/hal-03379142}

Submitted on 14 Oct 2021

HAL is a multi-disciplinary open access archive for the deposit and dissemination of scientific research documents, whether they are published or not. The documents may come from teaching and research institutions in France or abroad, or from public or private research centers.
L'archive ouverte pluridisciplinaire HAL, est destinée au dépôt et à la diffusion de documents scientifiques de niveau recherche, publiés ou non, émanant des établissements d'enseignement et de recherche français ou étrangers, des laboratoires publics ou privés. 
4

\section{Supramolecular organization and biological interaction of the squalenoyl siRNA} nanoparticles (1)

Marie Caillaud, ${ }^{1}$ Frédéric Gobeaux ${ }^{2}$, Miryana Hémadi ${ }^{3}$, Suzan Boutary ${ }^{1}$, Patrick Guenoun ${ }^{2}$, Didier Desmaële ${ }^{4}$, Patrick Couvreur ${ }^{4}$, Frank Wien ${ }^{5}$, Fabienne Testard ${ }^{2}$ and, Liliane MassaadMassade $^{1}$ 8

${ }^{1}$ U1195 Diseases and Hormones of the Nervous System, INSERM U1195 and University Paris-Saclay, 94276, Le Kremlin-Bicêtre, France.

${ }^{2}$ LIONS - NIMBE CEA, CNRS, Université Paris-Saclay, CEA Saclay, 91191 Gif-sur-Yvette Cedex, France.

${ }^{3}$ Université de Paris, CNRS-UMR 7086, Interfaces, Traitements, Organisation et DYnamique des Systèmes (ITODYS), UFR de Chimie 75013 Paris, France.

${ }^{4}$ Institut Galien Paris-Sud, CNRS UMR 8612, Université Paris-Sud, Université Paris-Saclay, 92290 Châtenay-Malabry, France.

${ }^{5}$ SOLEIL Synchrotron, Saint Aubin, France.

*Corresponding author: Liliane Massade, PhD, INSERM U1195, 94276 Le KremlinBicêtre, France. Email: liliane.massade@ inserm.fr, Phone: + 33149591830 1

Funding: This work is supported by a public grant overseen by the French National Research Agency (ANR) as part of the "Investissements d'Avenir" program (Labex NanoSaclay, reference: ANR-10-LABX-0035).

Key words: siRNA, nanotechnologies, structural studies, biological interaction

Chemical keywords: Azido-Squalene $\left(\mathrm{N}_{3}-\mathrm{SQ}\right)$, cationic squalenaldehyde hydrazinoguanidinium acetate salt $\left(\mathrm{SQ}^{+}\right)$, dibenzylcyclooctyne (DBCO), $\mathrm{N}$-(hexamethylenyl)6-oxohexanamide spacer (C6), Bovine serum albumin (BSA), Low density lipoprotein (LDL),

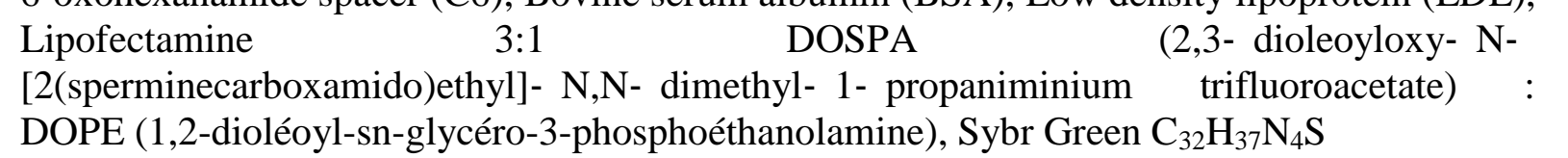


$\underline{\text { Table of contents }}$

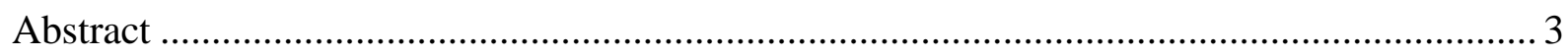

1. Introduction

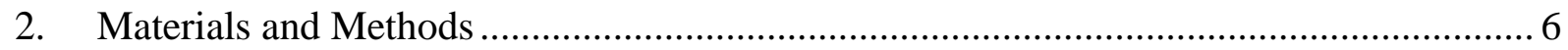

2.1. Chemicals

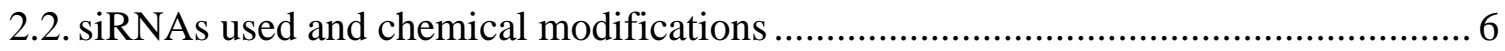

2.3. Preparation of siRNA-SQ nanoparticles........................................................... 7

2.4. Physicochemical characterization of siRNA-SQ nanoparticles ............................... 7

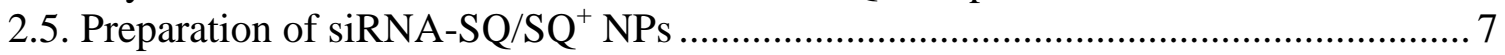

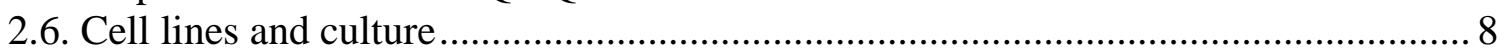

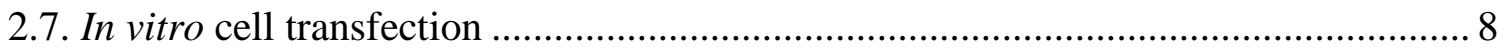

2.8. mRNA Extraction and Real-Time PCR (RT-qPCR) experiments ........................... 8

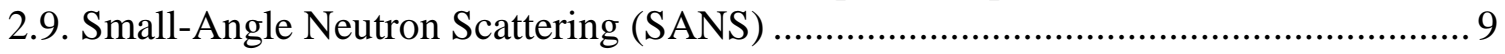

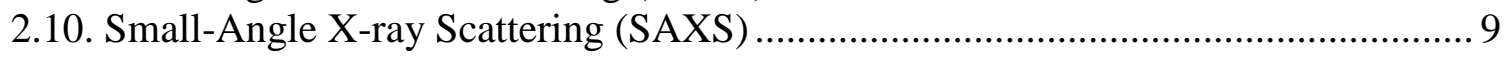

2.11. siRNA-SQ NPs internal structure characterization .............................................. 9

2.12. Electrophoresis of siRNA PMP22-SQ NPs incubated in FBS or with serum

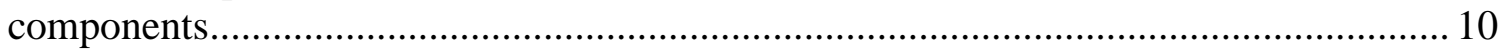

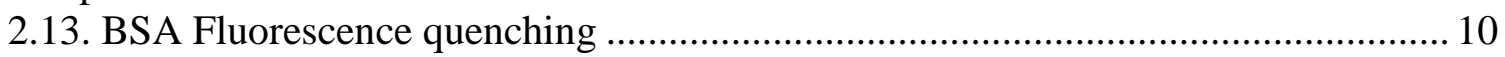

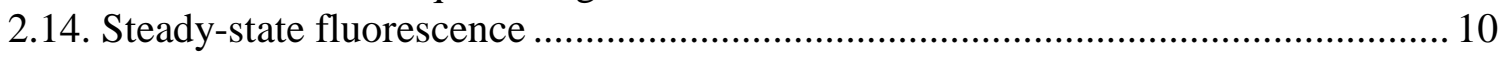

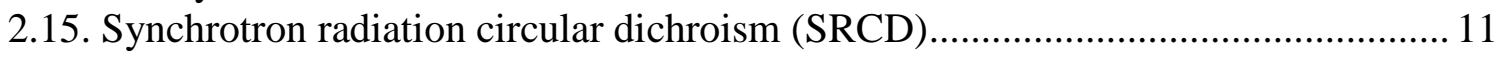

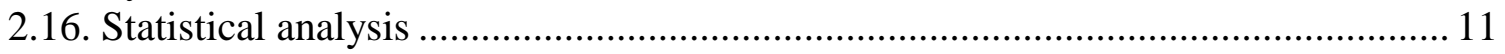

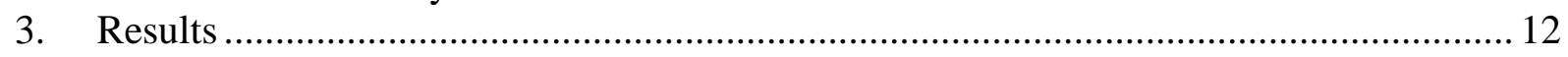

3.1. Structure determination of siRNA-SQ NPs ...................................................... 12

3.1.1. The physico-chemical characteristics of squalenoyl siRNA nanoparticles are

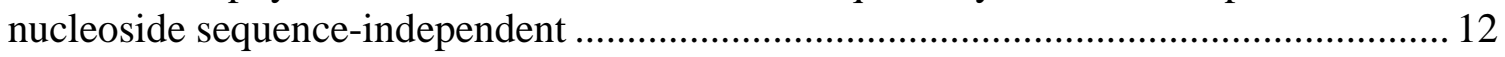

3.1.2. Inversion from negative to positive charge of siRNA-SQ NPs improves their

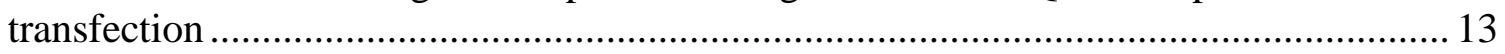

3.1.3 Spherical nanoparticles contain core/shell assemblies........................................ 14

3.1.4. A $130 \mathrm{~nm}$ nanoparticle can encapsulate around 60000 siRNAs ........................ 17

3.2. siRNA PMP22-SQ NP interactions with serum and its components....................... 19

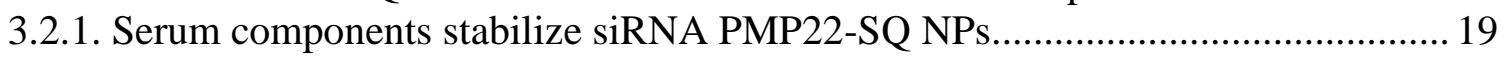

3.2.2. Serum neutralizes the siRNA-SQ NPs surface charge ...................................... 20

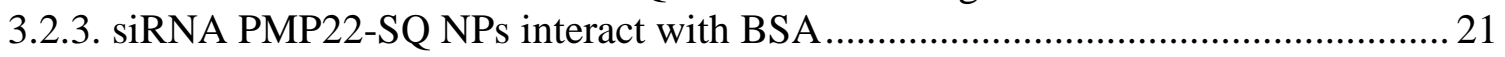

3.2.4. siRNA-SQ NPs interact with LDL.......................................................... 24

3.2.5. siRNA PMP22-SQ NPs interact via the squalene moiety with serum components 24

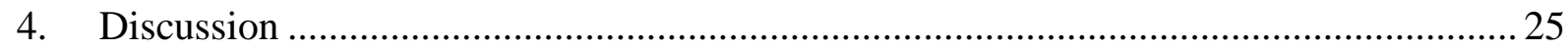

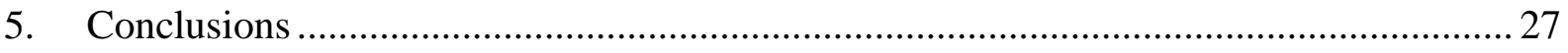

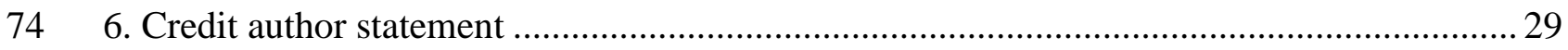

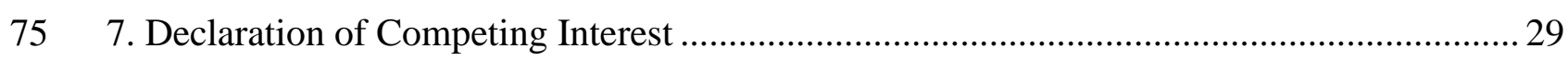

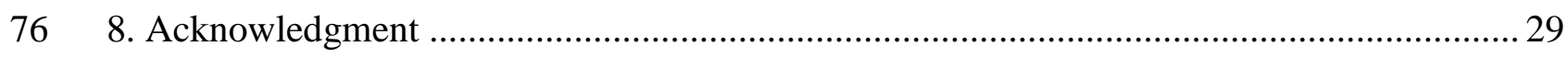

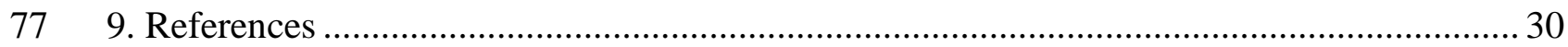

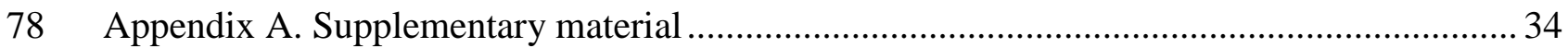


Small interfering RNAs (siRNA) are attractive and powerful tools to inhibit the expression of a targeted gene. However, their extreme hydrophilicities combined with a negative charge and short plasma half-life counteract their use as therapeutics. Previously, we chemically linked siRNA to squalene (SQ) which self-assembled as nanoparticles (NPs) with pharmacological efficiency in cancers and recently in a hereditary neuropathy. In order to understand the siRNA-SQ NP assembly and fate once intravenously injected, the present study detailed characterization of siRNA-SQ NP structure and its interaction with serum components.

We discovered that the siRNA-SQ bioconjugate self-assembled as 11-nm diameter core/shell supramolecular assemblies, which are connected one to another to form spherical nanoparticles of around 130-nm diameter. The siRNA-SQ NPs were stable in biological media and interacted with serum components, notably with albumin and LDL. The high specificity of siRNA to decrease or normalize gene expression and the high colloidal stability when encapsulated into squalene nanoparticles offer promising targeted therapy with wide applications for pathologies with gene expression dysregulation.

\section{Introduction}

Small interfering RNAs (siRNA) discovered by Fire and Mello at the end of the 90s (Fire, Xu et al. 1998), are small double-stranded RNAs composed of 19-21 bases pairs with 3'-cohesive ends. The antisense strand is complementary to a targeted messenger RNA (mRNA), leading to its degradation and gene silencing (Elbashir 2001, Elbashir, Martinez et al. 2001). Despite their specificity and efficiency at low doses, their intrinsic parameters constitute the main hurdles for in vivo delivery (Caillaud, El Madani et al. 2020). Indeed, in blood, naked siRNAs are rapidly degraded by nucleases and cleared by glomerular filtration, resulting in short plasmatic half-life (less than 15 mins) (Thompson, Kornbrust et al. 2012, Ozpolat, Sood et al. 2014). Moreover, their hydrophilic and polyanionic nature prevents efficient cellular uptake (Whitehead, Langer et al. 2009, Kanasty, Dorkin et al. 2013).

We previously developed an original approach for the conjugation of siRNA to squalene (SQ), a precursor of the cholesterol's biosynthesis. The so-called "Squalenoylation" technology consists of the bioconjugation of this lipid to a biologically active molecule, adopting a compact molecular conformation in aqueous media (Couvreur, Stella et al. 2006, 
112 Couvreur, Reddy et al. 2008, Desmaele, Gref et al. 2012). Squalene was initially covalently 113 coupled to siRNA via maleimide-sulfhydryl chemistry and the resulting siRNA-SQ 114 nanoparticles (siRNA-SQ NPs) showed significant results in reducing tumor growth in two 115 cancer models with fusion oncogenes: RET/PTC1 and RET/PTC3 (present in papillary 116 thyroid carcinoma) and TMPRSS2-ERG (found in prostate cancer) (Raouane, Desmaele et al. 117 2011, Ali, Maksimenko et al. 2014, Ali, Urbinati et al. 2014, Urbinati, Ali et al. 2015, 118 Urbinati, de Waziers et al. 2016). The conjugation method has been further improved, 119 implementing strain-promoted azide-alkyne cycloaddition. This copper-free (Cu-free) click chemistry approach increased the bioconjugation yield while preserving the antineoplastic activity of siRNA TMPRSS2-ERG-SQ NPs in a xenografted cancer model (MassaadMassade, Boutary et al. 2018). Recently, we succeeded in delivering squalenoyl siRNA targeting PMP22, an overexpressed gene causing hereditary neuropathy, i.e. the CharcotMarie-Tooth 1A (CMT1A) disease. The intravenous administration of siRNA PMP22-SQ NPs restored both motor and neuromuscular activities in transgenic mouse models (Boutary, Caillaud et al. 2021). Moreover, the targeted gene expression normalization indicated that the NPs successfully entered into the targeted tissue, the sciatic nerve and more specifically inside Schwann cells.

To better understand the siRNA-SQ NPs fate, their structure, as well as their biological behavior once intravenously injected into the circulating bloodstream should be investigated. Indeed, characterization of size, shape, surface properties and structure of siRNA-SQ NPs is important because these parameters may dramatically affect their biological outcome such as tissue accumulation and cell uptake (Kang, Kim et al. 2016, Dormont, Rouquette et al. 2019). In our previous studies, basic physicochemical parameters, like overall size and surface charge, were determined. However, their supramolecular organization, another key issue for the therapeutic efficiency in terms of availability and release of the active molecule (Lepeltier, Bourgaux et al. 2014, Kang, Kim et al. 2016), had never been investigated. Therefore, in the present study, high-resolution small-angle scattering has been performed to obtain new insights on the supramolecular organization of these nanoparticles.

In vivo interactions of nanoparticles with blood components represent another key point for biomedical applications. The interaction with serum biomolecules has already been investigated with squalenoyl conjugates bearing a small active functional group, like gemcitabine, doxorubicin (Sobot, Mura et al. 2017, Sobot, Mura et al. 2017, Yesylevskyy, Ramseyer et al. 2018) or adenosine (Gobeaux, Bizeau et al. 2020) but, never so far with macromolecules such as siRNA. Consequently, understanding the interaction between 
146 siRNA-SQ NPs and blood proteins is mandatory for further development towards clinical

147 applications. Among serum components interacting with NPs, we have focused our attention 148 on albumin and lipoproteins (LPs).

149 Albumin is the most abundant serum protein (55\%) and acts as carrier for several other 150 endogenous proteins, such as steroids and fatty acids (Sleep 2015, Mariam, Sivakami et al. 151 2016, Lamichhane and Lee 2020). Human albumin has been investigated and approved in 152 clinics to deliver paclitaxel, a chemotherapy drug, to cancer cells (Abraxane $\AA$ ) or to extend 153 the plasmatic half-life of insulin hormone linked to a fatty acid (Levemir®), which allows for $15498 \%$ binding to this protein (Kratz 2008). On the other hand, lipoproteins (LPs) are the third 155 most abundant plasma molecules in humans. They consist of an assembly of lipids and 156 proteins. Low-density lipoproteins (LDL) are the main carriers for cholesterol (75\%) and fatty 157 acids in mammals (Schumaker and Adams 1969). Thanks to their apolipoproteins 158 (amphiphilic protein) embedded in the outer membrane, LPs bind to lipoprotein receptors 159 resulting in cellular uptake by clathrin-mediated endocytosis (Chaudhary, Bower et al. 2019).

160 These macromolecules interact in the bloodstream with lipophilic drugs and deliver them to 161 tissues, such as the liver and tumors (Wolfrum, Shi et al. 2007, Wasan, Brocks et al. 2008, 162 Chaudhary, Bower et al. 2019).

163 In the present study, we first fully characterized the external and internal structure of 164 siRNA-SQ NPs at the supramolecular level and we assessed the interactions between siRNA165 SQ NPs and serum components, notably albumin and LDL. 
All chemicals used were of highest analytical grade and purchased from Sigma-Aldrich. The anhydrous solvents were dried and distilled before use (tetrahydrofuran (THF) on $\mathrm{Na}$ /benzophenone) and reactions sensitive to air or humidity were performed under nitrogen pressure. $\mathrm{D}_{2} \mathrm{O}(99.85 \%$

D) for small angle neutron scattering (SANS) experiments was purchased from Eurisotop (France).

175 Modified siRNAs for the $\mathrm{Cu}$-free click chemistry reactions were purchased from Eurogentec

176 (France). Azido-Squalene and cationic squalenaldehyde hydrazinoguanidinium acetate salt $\left(\mathrm{SQ}^{+}\right)$were synthetized according to our previous studies (Bertrand, Lucas et al. 2015, Massaad-Massade, Boutary et al. 2018). Dulbecco's modified Eagle medium (DMEM), OptiMEM, fetal bovine serum (FBS) were purchased from Fisher Scientific (Illkirch, France). UltraPure $^{\mathrm{TM}}$ distilled water (Dnase and Rnase free water) purchased from Invitrogen. Water was purified using a Milli-Q system. LDLs from human plasma [dissolved in $10 \mathrm{mM}$ Tris, $150 \mathrm{mM} \mathrm{NaCl}, 0.3 \mathrm{mM}$ EDTA pH 8.3 and $2 \mathrm{mM} \mathrm{NaN}_{3}$ ] were purchased from Invitrogen (Thermo Fisher Scientific). For small-angle scattering and fluorescence experiments, lyophilized BSA powder was dissolved to a $200 \mu \mathrm{M}$ concentration in $0.2 \mathrm{M}$ Phosphate Buffer Saline (PBS) then in $\mathrm{H}_{2} \mathrm{O}$ for standard experiments and in $\mathrm{D}_{2} \mathrm{O}$ for SANS experiments. BSA solutions were dialyzed overnight against at least a 10-fold volume $\mathrm{PBS} / \mathrm{D}_{2} \mathrm{O}$ (or $\mathrm{PBS} / \mathrm{H}_{2} \mathrm{O}$ ) solution, then stored at $4{ }^{\circ} \mathrm{C}$. FBS was also dialyzed overnight against $\mathrm{PBS} / \mathrm{D}_{2} \mathrm{O}$ just after bottle opening.

\section{2. siRNAs used and chemical modifications}

The siRNA targeting RET/PTC1 and PMP22 used in the experiments were previously described (Ali, Maksimenko et al. 2014, Massaad-Massade, Boutary et al. 2018, Boutary,

193 Caillaud et al. 2021). All single-stranded RNAs were synthesized by Eurogentec (France) and

194 purified by RP-HPLC. Single-strand RNAs were synthesized as 19-mers with two 3'overhanging 2'-deoxynucleotide residues to provide stabilization against nucleases, as 196 described by Tuschl et al. (Tuschl 2002). To allow conjugation with squalene, a 197 dibenzocyclooctyne (DBCO) reactive group and a linker N-(hexamethylenyl)-6198 oxohexanamide spacer (C6) were introduced at the 5'-end of the sense strand of each siRNA 199 (Massaad-Massade, Boutary et al. 2018). 
To anneal naked siRNA (i.e. siRNA not coupled to SQ), equimolar amounts of sense and antisense strands were mixed with the hybridization buffer [30 mM HEPES-KOH (pH 7.4), 2 $\mathrm{mM} \mathrm{Mg}$ acetate, $100 \mathrm{mM} \mathrm{K}$ acetate]. The mixture was incubated for $3 \mathrm{~min}$ at $95^{\circ} \mathrm{C}$ and then for $45 \mathrm{~min}$ at room temperature before being stored at $-80^{\circ} \mathrm{C}$.

\subsection{Preparation of siRNA-SQ nanoparticles}

$\mathrm{Cu}$-free click chemistry, a 1,3-dipolar cycloaddition was developed by our lab as previously described (Massaad-Massade, Boutary et al. 2018, Boutary, Caillaud et al. 2021). Then, the siRNA-SQ NPs were prepared by inverse nanoprecipitation in acetone/water as sketched in

Figure S1. Practically, annealed siRNA-SQ bioconjugates in UltraPure ${ }^{\mathrm{TM}}$ distilled water (Dnase and Rnase free water) were added drop wisely to a stirred organic phase (acetone) with a volume/volume ratio of 1 [organic phase]: 2 [aqueous phase]. Then acetone was evaporated using nitrogen flux for 30 min to obtain an aqueous suspension of pure siRNA-SQ 213 NPs.

\subsection{Physicochemical characterization of siRNA-SQ nanoparticles}

Hydrodynamic diameter, PDI and $\zeta$-potential were measured by dynamic light scattering (DLS) using the Malvern Zetasizer Nano ZSP (Malvern Instrument) with a scattering angle of $173^{\circ}$ for size measurements. Samples were analyzed at $10 \mu \mathrm{M}$ concentration in RNase-free water.

221 Cryogenic Transmission Electron Microscopy (cryo-TEM) was performed with a JEOL 2100 electron microscope at the Electronic Microscopy Platform (IBPS/Institut de Biologie ParisSeine, Université P. et M. Curie, Paris, France). A $4 \mu \mathrm{L}$ drop of the concentrated siRNA-SQ NPs $(166 \mu \mathrm{M})$ was deposited on a carbon-coated copper grid. Excess liquid was removed with blotting filter paper, and the samples were quickly vitrified by plunging them into liquid ethane using a guillotine-like frame. The samples were then transferred to a cryo-sample holder. Observations were made at an acceleration voltage of $200 \mathrm{kV}$ under a low electron dose. Analysis was performed with Image J software.

\subsection{Preparation of siRNA-SQ/SQ ${ }^{+}$NPs}

231 Cationic squalenaldehyde hydrazinoguanidinium acetate salt $\left(\mathrm{SQ}^{+}\right)$was dissolved in acetone 232 at a concentration of $1 \mathrm{mg} / \mathrm{mL}$ and added dropwisely to a suspension of already prepared 233 siRNA-SQ NPs at a molar ratio of $1[\mathrm{NPs}]: 100\left[\mathrm{SQ}^{+}\right]$. The organic solvent was then 
evaporated for 30 min under stirring using the nitrogen flow to obtain siRNA-SQ/SQ ${ }^{+} \mathrm{NPs}$ in aqueous solution. Then size and $\zeta$-potential were measured.

\subsection{Cell lines and culture}

BHP 10-3 is a papillary thyroid carcinoma cell line harboring the fusion oncogene RET/PTC1 and MSC80 is a mouse Schwann cell line expressing PMP22. Both cell lines were maintained at $37{ }^{\circ} \mathrm{C}$ in an atmosphere of $5 \% \mathrm{CO}_{2}$ and $95 \%$ humidity in complete DMEM supplemented with $10 \%$ heat-inactivated FBS, with 100 units $/ \mathrm{mL}$ penicillin, and $100 \mu \mathrm{g} / \mathrm{mL}$ streptomycin.

\subsection{In vitro cell transfection}

One feature of siRNA-SQ NPs is that they cannot spontaneously enter into cells in vitro without the help of a transfection agent (Raouane, Desmaele et al. 2011, Ali, Maksimenko et al. 2014, Urbinati, Ali et al. 2015, Massaad-Massade, Boutary et al. 2018, Boutary, Caillaud et al. 2021). Therefore, Lipofectamine 2000 was used to assess naked siRNA and siRNA-SQ NPs efficiency in vitro.

To study the effect of surface charge, $3 \times 10^{5}$ cells of BHP 10-3 and MSC80 were seeded, 24 $\mathrm{h}$ before transfection, in six-well plates in DMEM supplemented with 10\% FBS, penicillin (100 U/mL), and streptomycin $(10 \mu \mathrm{g} / \mathrm{mL})$. The next day, the cell medium was replaced with 2mL of Opti-MEM medium (Gibco Life technologies, ref. 11058021). The siRNA-SQ NPs and naked siRNA were transfected using Lipofectamine 2000, according to the supplier's recommendations, in the reduced Opti-MEM medium for a final concentration of $50 \mathrm{nM} / \mathrm{well}$. Similarly, siRNA-SQ/SQ ${ }^{+}$NPs and siRNA-SQ NPs alone (as a negative control) were added to Opti-MEM and incubated with the cells. 4h later, the medium was replaced by complete DMEM and $48 \mathrm{~h}$ later the experiments were stopped, and RNA was extracted to analyze silencing of targeted genes RET/PTC1 and PMP22. Each experiment was performed at least twice in duplicate.

\section{8. mRNA Extraction and Real-Time PCR (RT-qPCR) experiments}

Total RNA was extracted from BHP 10-3 and MSC80 cells using the RNeasy mini-kit (Qiagen, Courtaboeuf, France). Reverse transcription was performed using the M-MLV RT buffer pack (Invitrogen, Charbonnières-les-Bains, France). Then, real-time PCR (qPCR) was carried out with the CFX96TM Real-time system (Biorad) using the Maxima Syber Green Rox qPCR master Mix (Thermo Scientific, Villebon-sur-Yvette, France), according to the manufacturer's instructions. Samples were run in duplicate; gene expression was determined 
by the $2^{-\Delta \Delta C t}$ method. Relative mRNA levels of targeted genes (RET/PTC1 and PMP22) were compared to non-treated (NT) cells after normalization regarding control gene levels in at least 2 independent experiments.

\subsection{Small-Angle Neutron Scattering (SANS)}

SANS experiments were carried out on the D33 spectrometer at ILL (doi: 10.5291/ILLDATA.9-13-781). Samples prepared in $\mathrm{D}_{2} \mathrm{O}$ were placed in 1-mm size Hellma quartz cells and thermostated at $20^{\circ} \mathrm{C}$. The raw data were radially averaged, corrected for electronic background and normalized by water scattering using the LAMP software (Richard, Ferrand et al. 1996). Three configurations were used to cover a $q$ range going from 0.0013 to $0.27 \AA^{-1}$ : 1) wavelength: $\lambda=13 \AA$, sample detector distance $\mathrm{D}=12 \mathrm{~m}$, collimation: $\Phi=12.8,2) \lambda=4.6$ $\AA, \mathrm{D}=12 \mathrm{~m}$, collimation: $\Phi=7.8$ and 3) $\lambda=4.6 \AA, \mathrm{D}=2 \mathrm{~m}$, collimation $\Phi=7.8$, for small, medium and large angles, respectively.

\subsection{Small-Angle X-ray Scattering (SAXS)}

Small-angle X-ray scattering patterns (SAXS) were obtained on the SWING beamline of the SOLEIL synchrotron (Saint-Aubin, France). The scattering vector range $q$ of $2 \times 10^{-3} \AA^{-1}$ to 2.5 $\AA^{-1}$ was obtained with an energy of $16 \mathrm{keV}$ and two sample-to-detector distances (6 and 0.50 $\mathrm{m}$ for the SAXS and the wide-angle X-ray scattering ranges, respectively). The data were recorded by a two-dimensional Eiger 4M (Dectris) detector. Data reduction was performed using Foxtrot software, an application developed at SOLEIL. The sample was placed in a 1.5$\mathrm{mm}$ diameter quartz capillary sealed with kerosene wax.

\subsection{1. siRNA-SQ NPs internal structure characterization}

Analysis of the SANS and SAXS patterns of siRNA PMP22-SQ NPs in aqueous solution allows to draw a first sketch of the shape and internal structure of the NPs. The scattering curves were fitted by SASview software (Www.sasview.org/) to a lognormal sphere distribution model for the siRNA-SQ particles and a cylinder model for the siRNA moiety. The fitting of the experimental curves of siRNA led to a cylindrical shape whose volume was given by the following formula: $\mathrm{V}(\mathrm{siRNA})=\pi \times$ radius $^{2} \times$ height. Fitting of the curves of siRNA-SQ NPs gave a spherical distribution. The nanoparticle volume corresponded to: (4/3) $\mathrm{x} \pi \mathrm{x}$ radius $^{3}$. Consequently, the ratio between the volume of the siRNA-SQ nanoparticle and the volume of the siRNA made it possible to determine the number of siRNAs linked to SQ in a nanoparticle. 


\subsection{Electrophoresis of siRNA PMP22-SQ NPs incubated in FBS or with serum components}

Interactions between siRNA PMP22-SQ NPs and FBS or with one of its main components (BSA or LDL) were performed by incubating increasing amounts of FBS, BSA (molar ratio) or LDL (mass ratio) with $5 \mu \mathrm{M}$ siRNA PMP22-SQ NPs for $30 \mathrm{~min}$ at $37^{\circ} \mathrm{C}$. The same volume of each aliquot was loaded into $4 \%$ agarose electrophoresis gel stained with Midori Green Advance (Nippon Genetic Europe, Germany) that only reveals the oligonucleotides. The molecular weight (MW) size marker served as an evidence to testify the migration of siRNA PMP22-SQ NPs. Bands were revealed by camera under UV lamp illumination and recorded by Visio-capt 14.2 software.

\subsection{BSA Fluorescence quenching}

A series of BSA solutions at a fixed concentration $(5 \mu \mathrm{M})$ incubated with an increasing amount of siRNA PMP22-SQ NPs (BSA/siRNA PMP22-SQ molar ratios $=0.5,1,2,4$ and 8 ) were analyzed. Solutions were filled into Hellma quartz Ultra-Micro cells and placed in a Cary Eclipse fluorimeter (Varian, Oxford, UK) for measurements. The samples were excited at $280 \mathrm{~nm}$ and emission scans were recorded between 300 and $550 \mathrm{~nm}$. Spectra were averaged over 5 measurements. Quenching experiments could affected by the inner filter effect, where the sample could attenuate both the excitation beam and the emission spectrum. However, in our case, we worked with rather low concentrations of both BSA and siRNA PMP22-SQ NPs, and the optical density of the solutions was below 0.1 ; thus, this effect could be ruled out.

\subsection{Steady-state fluorescence}

To a fixed concentration of siRNA PMP22-SQ NPs $(5 \mu \mathrm{M}$, i.e. $0.065 \mathrm{mg} / \mathrm{mL})$, the BSA (100 $\mu \mathrm{M}$ stock solution) or LDL (2.5 mg/mL stock solution) were gradually added to achieve a final molar ratio of 1 [siRNA PMP22-SQ]:1 [BSA $5 \mu \mathrm{M}$ ] or mass ratio of 1 [siRNA PMP22SQ]:2 [LDL $0.12 \mathrm{mg} / \mathrm{mL}$ ]. Briefly, $1 \mathrm{~mL}$ of a $5 \mu \mathrm{M}$ or $0.065 \mathrm{mg} / \mathrm{mL}$ siRNA PMP22-SQ NPs solution was filled into a Hellma Ultra-Micro quartz cell and $2 \mu \mathrm{L}$ of BSA or LDL were progressively added until reaching the final ratio. In parallel, BSA and LDL were added to water to reach the same final concentration. After each addition, samples were excited at 280 
$\mathrm{nm}$ and the fluorescence emission spectra were recorded from 300 to $400 \mathrm{~nm}$ using a Horiba

337 Jobin Yvon Fluorolog 3 spectrometer.

\section{$\underline{\text { 2.15. Synchrotron radiation circular dichroism (SRCD) }}$}

340 Synchrotron radiation circular dichroism (SRCD) experiments were performed on the DISCO 341 beamline at the SOLEIL Synchrotron (Saint Aubin, France). The conformation of naked 342 siRNA PMP22 at two stages of NPs preparation (before and after the nanoprecipitation) was 343 performed, as well as, the study of the interaction with BSA by mixing siRNA PMP22-SQ 344 NPs and BSA at a volume/volume ratio of 1 [siRNA PMP22-SQ]:1 [BSA]. Four $\mu$ L droplets 345 of the samples were placed in $3.7 \mu \mathrm{m}$ path length $\mathrm{CaF}_{2}$ cells (Hellma). The raw spectra were 346 acquired with a $1 \mathrm{~nm}$ spectral resolution. The spectra presented in this study were treated with 347 CDTool software that can be freely downloaded at http://www.cdtools.cryst.bbk.ac.uk/ (Miles 348 and Wallace 2018). Each is the average of three spectra. A background (water or 349 corresponding buffer spectrum recorded under the same conditions) was subtracted. Intensity 350 calibration was obtained with a camphor sulfonic acid CSA scale standard sample. Intensities 351 were converted from millidegrees ( $\theta$ machine units) into $\Delta \varepsilon$ using the formula $\Delta \varepsilon=$ $352 \theta \times(0.1 \times \mathrm{MRW}) /\left(3298 \times l^{*} C\right)$, where MRW is the mean residue weight of the BSA (protein 353 weight/number of residues $=114 \mathrm{Da}$ ), $l$ is the path length of the $\mathrm{CaF}_{2}$ cell in $\mathrm{cm}$ and $C$ is the 354 protein concentration in $\mathrm{mg} / \mathrm{ml}$. In some cases, we have added a smoothing function.

\subsection{Statistical analysis}

358 Statistics were computed with GraphPad Prism 8.3.0 software. When we had two groups to compare, the Mann-Whitney analysis was performed to assess the statistical difference. For studies requiring grouped analyses, a one-way ANOVA followed by Bonferroni multiple comparison test was performed. A value of $p<0.05$ was considered significant. 


\subsection{Structure determination of siRNA-SQ NPs}

3.1.1. The physico-chemical characteristics of squalenoyl siRNA nanoparticles are nucleoside sequence-independent

To obtain nanoparticles, an inverse nanoprecipitation method (Bilati, Allémann et al. 2005) was performed by the addition of the aqueous solution containing the siRNA-SQ bioconjugates into acetone as an organic solvent, where siRNA-SQs were not soluble. The size and polydispersity of two resulting nanoformulations with siRNA RET/PTC1 or siRNA PMP22 were measured by DLS (Figures 1A and 1B). For the siRNA RET/PTC1, conjugation to SQ led to $180 \mathrm{~nm}$ NPs with a homogeneous distribution as indicated by the polydispersity index (PDI; less than 0.2). Surface charge of NPs was negative, around -35 $\mathrm{mV}$, which favors colloidal stability of the NP suspension (Figure 1C). For siRNA PMP22, the results were similar: NPs size was $218 \mathrm{~nm}$, PDI $<0.2$ and surface charge $-40 \mathrm{mV}$. No significant difference was observed between siRNA RET/PTC1-SQ and siRNA PMP22-SQ NPs in terms of size, polydispersity index and $\zeta$-potential (Figures 1A, 1B and 1C). As no significant difference was found concerning the physicochemical parameters of both siRNAs (PMP22 or RET/PTC1) and previously with the siRNA TMPRSS2-ERG (Massaad-Massade,

381 Boutary et al. 2018), it was decided to continue further experiments with the siRNA PMP22-

382 SQ NPs. This resolution is also supported by our recent findings showing that the siRNA 383 PMP22-SQ NPs could lead to a targeted therapy for CMT1A neuropathy (Boutary, Caillaud et al. 2021).

Then, the siRNA PM22-SQ NPs were observed by cryo-TEM (Figure 1D). Spherical nano-objects of around $130 \mathrm{~nm}$ in size were observed. Interestingly, the shape and size were in accordance with our previous observations (Massaad-Massade, Boutary et al. 2018). 

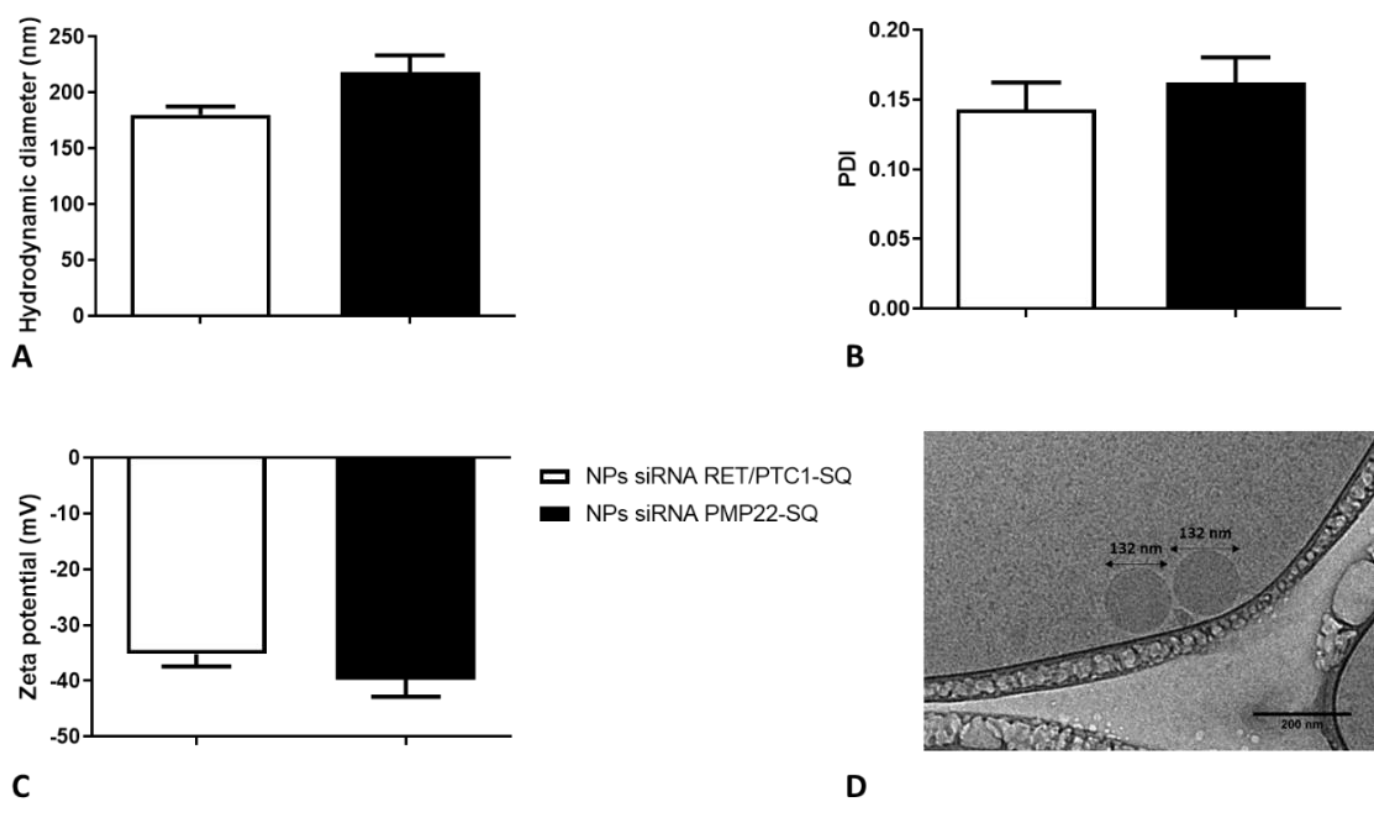

Figure 1: Physicochemical characterization of siRNA-SQ NPs. After inverse nanoprecipitation of siRNA RET/PTC1-SQ NPs and siRNA PMP22-SQ NPs the size (A) and polydispersity index (B) were measured by dynamic light scattering and the surface charge (C) by electrophoretic light scattering. Three measurements were performed and the average diameter \pm standard error of the mean (SEM) of more than 5 independent nanoformulations, was calculated. Using the Mann and Whitney test, no significant difference between the two nanoformulations was found. D) Micrography of siRNA PMP22-SQ NPs by cryoTEM. The siRNA-SQ NPs are spherical with a diameter of around $130 \mathrm{~nm}$.

3.1.2. Inversion from negative to positive charge of siRNA-SQ NPs improves their transfection

To understand the impact of surface charge on the internalization of siRNA-SQ NPs, several experiments were conducted in vitro. Previously, we showed that the siRNA-SQ NPs were unable to enter cultured cells spontaneously without any cationic transfection agent (Raouane, Desmaele et al. 2011, Ali, Maksimenko et al. 2014, Boutary, Caillaud et al. 2021). We postulated that the negative surface charge of siRNA-SQ NPs prevented cell uptake due to electrostatic repulsion with the negative plasma membrane. It is well known that cationic nanoparticles had a higher internalization rate than neutral and negative nanoparticles. This difference can be explained by Coulomb's law, where opposite charges are attracted to each other. Thus, the cationic nanoparticles can be delivered to the cytoplasm by electrostatic attraction with the negatively charged cell membranes. To confirm this assumption, we modified the nanoparticle charge by addition of cationic guanidium-squalene to the siRNASQ NPs (Figure 2A). The resulting positive nanoparticles were then incubated with BHP 103 and MSC80, two cell lines of different origins (tumoral and normal). Interestingly, the 
B

A

inhibition of the targeted gene expression without using lipofectamine testified that the uptake of the nanoparticle is facilitated by the use of a cationic compound (Figures 2 B and 2C).

\begin{tabular}{|c|c|c|c|}
\hline & Size & PDI & Zeta potential \\
\hline siRNA-SQ NPs & $171.3 \pm 12.3 \mathrm{~nm}$ & $0.127 \pm 0.019$ & $-37.1 \pm 2.4 \mathrm{mV}$ \\
\hline siRNA-SQ NPs : SQ $^{+}$ & $225.5 \pm 8.5 \mathrm{~nm}$ & $0.119 \pm 0.014$ & $39.6 \pm 4.3 \mathrm{mV} * *$ \\
\hline
\end{tabular}
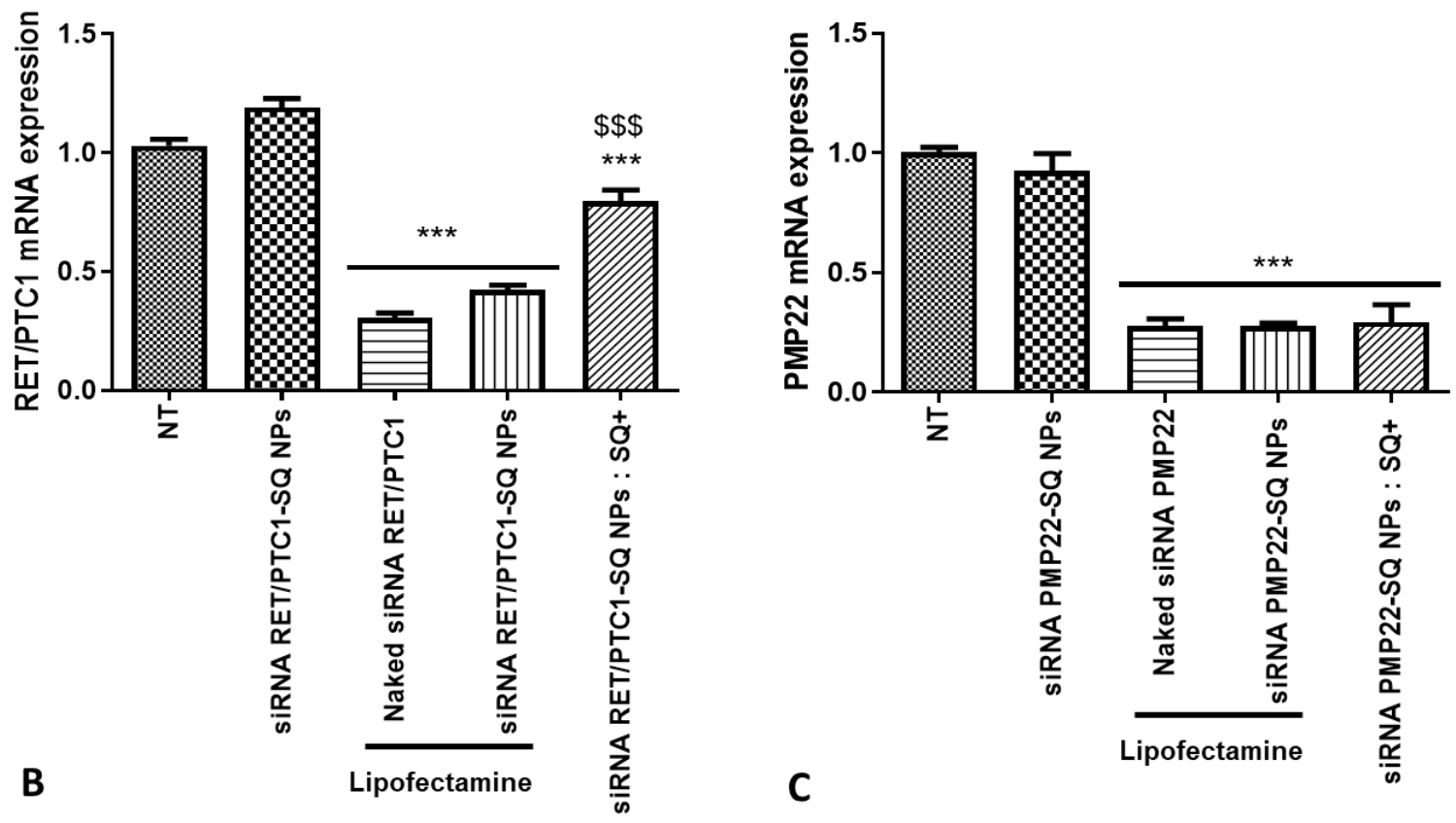

Figure 2: Positively charged siRNA-SQ NPs are successfully cell internalized. A) Physicochemical characteristics of NPs. No statistical difference was observed between siRNA-SQ NPs and siRNA-SQ NPs: $\mathrm{SQ}^{+}$for their size and PDI. Using the Mann-Whitney test, a statistical difference was found concerning the zeta potential between the siRNA-SQ NPs and siRNA-SQ NPs:SQ+ ( $<$ 0.001). B and C) mRNA inhibition. BHP 10-3 B) and MSC80 C) cells were transfected with $50 \mathrm{nM}$ siRNA RET/PTC1 or PMP22-SQ NPs with or without $\mathrm{SQ}^{+}$. NT: non-treated cells. Lipofectamine was also used to transfect the naked siRNA and siRNA RET/PTC1 or PMP22-SQ NPs (positive controls). 48 hours later, cells were harvested, RNA was extracted, and RT-qPCR performed. One-way Anova followed by a Bonferroni test was used to assess the significant difference between treatments: $* * * p<$ 0.0001 , compared to NT and untransfected targeted gene-SQ NPs ; $\$ \$ p<0.0001$ when compared to transfected naked siRNA RET/PTC1 and siRNA RET/PTC1-SQ NPs by lipofectamine.

\subsubsection{Spherical nanoparticles contain core/shell assemblies}

Based on our previous results showing impressive therapeutic results in several tumor models (thyroid and prostate cancers (Ali, Urbinati et al. 2012, Urbinati, Hafiz et al. 2013, Ali, Maksimenko et al. 2014, Ali, Urbinati et al. 2014, Urbinati, Ali et al. 2015, Urbinati, de Waziers et al. 2016) as well as in monogenic neuropathy (CMT1A (Boutary, Caillaud et al. 2021)) and considering that cationic lipid have a higher in vivo toxicity (Caillaud, El Madani et al. 2020), we decided to deeply characterize the conjugated siRNA-SQ nanoparticles. 
To verify that the conjugation to SQ and the formulation as nanoparticle did not induce changes in siRNA conformation, circular dichroism measurements were performed. As shown in Figure S2A, naked siRNA PMP22 and siRNA PMP22-SQ both before and after nanoprecipitation displayed the same spectrum type, confirming that the $\alpha$-helix structure of the siRNA was kept intact after chemical conjugation to SQ and formulation as nanoparticles. Small-angle scattering methods are prominent tools for characterizing such nanometric colloidal suspensions. Taking advantage of the high difference of the scattering length density between hydrogen and deuterium, small-angle neutron scattering (SANS) represents, indeed, a method of choice to characterize the size distribution of hydrogenated colloids in a deuterated solvent and it has been proven previously to be a good way of characterizing the size of the squalene-based nanoparticles (Saha, Testard et al. 2015, Rouquette, Ser-Le Roux et al. 2019, Gobeaux, Bizeau et al. 2020). On the other hand, small-angle X-ray scattering (SAXS), which is sensitive to contrasts in electron densities, offered a description of the squalenoyl siRNA nanoparticles with deeper access to their internal structure. In the case of siRNA PMP22-SQ NPs, it was relevant to combine SANS and SAXS analysis since the former would be capable of detecting a larger contrast between the solvent and the full squalene derivative, and the latter between the solvent and the siRNA moiety (Figure 3A). The SANS of a $45 \mu \mathrm{M}$ suspension of siRNA PMP22-SQ NPs is shown in Figure 3B. This pattern was not sensitive to dilution and the nanoparticle size remained stable under a dilution factor of two (Figure S2B). The absence of structural peaks in the wide-angle region $(q>$ $0.04 \AA^{-1}$ ) indicated that the nanoparticle core displayed no regular crystal-like structure, in accordance with cryo-TEM images (see Figure 1). In the medium $q$-range (0.006 to $0.02 \AA^{-1}$ ), the scattering intensity followed a power-law in $q^{-3.44}$ slightly deviating from the $q^{-4}$ expected for sharp interfaces (Porod's Law (Guinier, Fournet et al. 1955)) between particle surfaces and solvent. If such observation may eventually indicate a slightly diffuse or fractal interface, it was however unlikely, given that the siRNA PMP22-SQ NPs surface appeared rather smooth in the cryo-TEM images. At the lowest wavevectors, the scattering intensity reached the onset of a plateau indicating a finite size. A simple Guinier analysis gave a gyration radius $\left(R_{\mathrm{g}}\right)$ of $80 \mathrm{~nm}$ that corresponded to a hydrodynamic radius (as it would be determined by DLS) of $103 \mathrm{~nm}$ for a spherical shape. Overall, this scattering pattern could be fitted by considering a lognormal distribution of spheres (black line on red pattern in Figure 3B), with a median radius of $61.9 \mathrm{~nm}$ and a polydispersity index (PDI) of 0.25 . The corresponding diameter distribution resulting from this fit is presented in Figure 3C. 
The SAXS pattern of a $263 \mu \mathrm{M}$ solution of siRNA PMP22-SQ NPs (blue pattern) is presented in Figure 3D. Again, no specific structural peak could be observed in the large $q$ 472 region $\left(q>0.3 \AA^{-1}\right)$. On the contrary to the SANS pattern, two large oscillations were visible 473 in the middle $q$-range $\left(0.007<q<0.3 \AA^{-1}\right)$, that could be attributed to siRNA moieties in the 474 nanoparticle or at least to electronic density heterogeneities in the particle. As a matter of 475 comparison, the SAXS pattern of the siRNA PMP22 moiety $(224 \mu \mathrm{M})$ was also plotted (green 476 pattern). The scattering pattern originating from the naked siRNA PMP22 dispersion 477 exhibited an oscillation in the same $q$-range and could be fitted by a cylindrical form factor $(L$ $478=7.1 \mathrm{~nm}, R=1.1 \mathrm{~nm}$ ), in accordance with the SANS analysis of similar siRNA strands 479 reported by Ristori et al. (Ristori, Ciani et al. 2012) (Table S1).This characteristic shape was 480 found again in the oscillation, visible in the large $q$ of the siRNA PMP22-SQ pattern, 481 yielding the following dimensions: $L=4 \mathrm{~nm}$ and $R=1.1$ from the fitting by a cylindrical form 482 factor. The other oscillation (at lower $q$ ) could be fitted by spheres with a radius of $5.5 \mathrm{~nm}$. 483 Since these spheres could accommodate the siRNA strands, we suggested that the siRNA 484 PMP22 moieties were segregated and surrounded by the squalene moiety in core/shell 485 assemblies with interconnections of the squalene moieties within the NPs. The obtained 486 dimensions indicated a heterogeneity in electronic density inside the NPs, but without long 487 range ordering. 

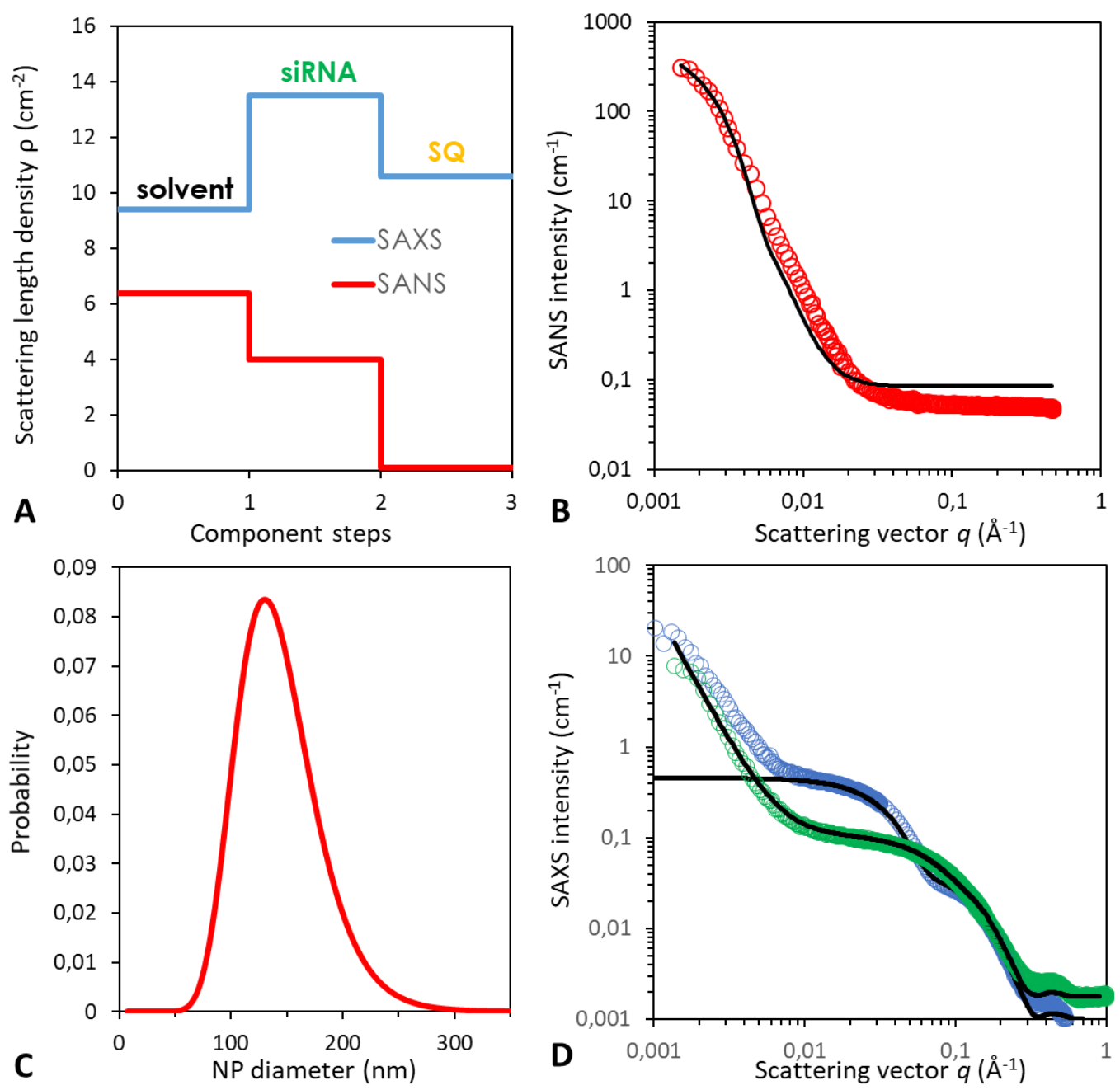

Figure 3: Structural characterization of siRNA PMP22-SQ NPs by small-angle scattering.

A) Scattering length densities show that the SANS approach allows to characterize the overall structure of the nanoparticles and the SAXS analysis to characterize the siRNA encapsulated within the NPs. The solvent used are $\mathrm{H}_{2} \mathrm{O}$ and $\mathrm{D}_{2} \mathrm{O}$ for SAXS and SANS, respectively. B) SANS pattern for siRNA PMP22-SQ NPs (open circles) depicting a fit with a lognormal distribution of spheres (black trace). C) SANS view software analysis of the fit B showed that the corresponding siRNA PMP22-SQ NPs diameters are $124 \mathrm{~nm}$. D) SAXS patterns of the naked siRNA PMP22 (green circles) and of siRNA PMP22-SQ NPs (blue circles) with their corresponding fits (black traces). The naked siRNA PMP22 can be fitted by a $7 \mathrm{~nm}$-long cylindrical shape and a scattering in $q^{-3}$ in the low $q$ range (a signature of aggregation). A combination of $4 \mathrm{~nm}$ cylindrical and $11 \mathrm{~nm}$ spherical shapes was found for the internal organization of the siRNA PMP22-SQ NPs.

\subsubsection{A $130 \mathrm{~nm}$ nanoparticle can encapsulate around 60000 siRNAs}

The structural model of siRNA PMP22-SQ NPs is illustrated by the sketch in Figure

4. The siRNA PMP22-SQ bioconjugate monomer is represented by a green cylinder for the siRNA moiety and a yellow lipid tail for the squalene moiety (Figure 4A). The 
supramolecular organization inside the nanoparticle is represented by $11-\mathrm{nm}$ core/shell

510 spheres with the lipidic moieties surrounding the hydrophilic siRNAs (Figure 4A). As no specific inner structure was apparent in the cryo-TEM images, the core/shell structures were supposed to be randomly distributed inside the whole nanoparticle volume. Therefore, representation of a top view nanoparticle cross-section depicts a random pattern of circular motifs (Figure 4B). Since the formation of squalene-based nanoparticles was clearly driven by hydrophobic interactions (Lepeltier, Bourgaux et al. 2014), we assumed that the squalene shells around the hydrophilic siRNA core could be interpenetrated and formed a continuous lipophilic network and it is most likely that some charged hydrophilic siRNA strands point towards the solvent (represented by the green circle around the yellow one in Figure 4B). By a simple geometrical calculation (volume of nanoparticle/volume of siRNA), we could estimate that one nanoparticle contained on average 60,000 siRNA PMP22-SQ bioconjugates.

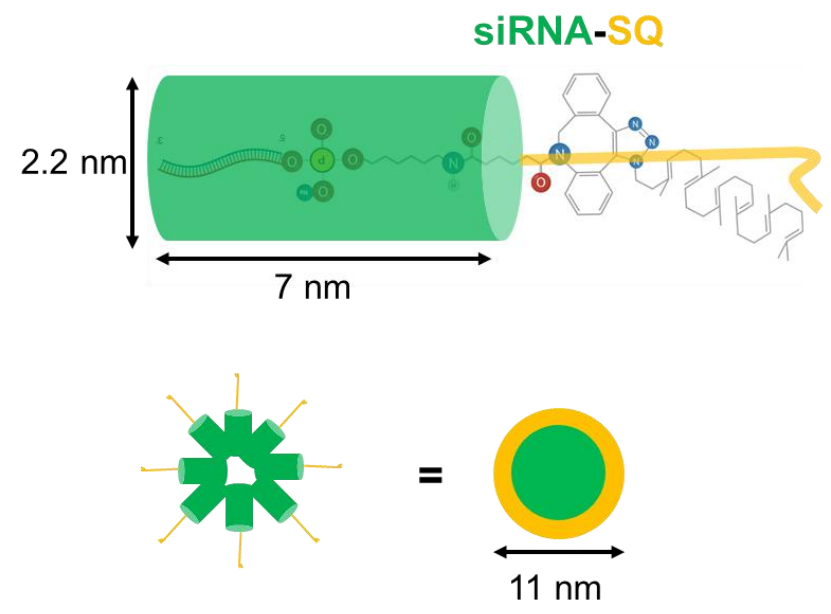

A

B

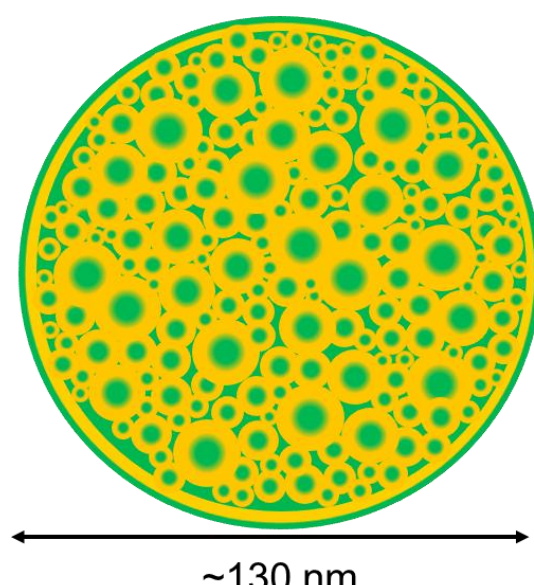

$\sim 130 \mathrm{~nm}$

Figure 4: Graphical representation of a siRNA PMP22-SQ nanoparticle as determined by the fit of the scattering patterns. A) Sketch of siRNA PMP22-SQ monomer. The siRNA moiety (green) of the bioconjugate can be viewed as a cylinder (siRNA not to scale in comparison to others chemical entities). The squalene moiety is depicted in yellow. The SAXS pattern reveals 11-nm spherical structures inside the nanoparticle that we view as selfassembled core/shell domains with a siRNA core surrounded by squalene which could interpenetrate with the other squalene moieties. B) Representation of a cross-section of 2D siRNA PMP22-SQ nanoparticle. The core/shell spheres of 11-nm each are randomly arranged inside the nanoparticle. The 2D cross section is the cause of the difference in the core/shell sphere size in the sketch. 
538 biological medium with high ionic strength containing several proteins, on the structure of 539 siRNA PMP22-SQ NPs. In the low $q$ range $\left(q<0.003 \AA^{-1}\right)$, the resulting SANS patterns did 540 not show strong deviation from the scattering intensity of the siRNA PMP22-SQ suspension 541 (at similar concentration) (Figure 5A). No intensity increase was observed, as it could be 542 expected when particles are growing or when attractive interactions appear between particles. 543 It demonstrates that siRNA PMP22-SQ NPs were colloidally stable and did not aggregate in 544 this complex mixture. On the contrary, the scattering intensity arising from the siRNA 545 PMP22-SQ NPs slightly decreased. As confirmed by the fitting curve (black line on Figure 546 5A), the SANS pattern of the mixture appeared to be a linear combination of the two 547 reference patterns. If we consider the formula: $I_{\text {fit }}=\mathrm{A} \times I$ single $(\text { siRNA PMP22-SQ NPs })_{\text {ini }}+$ $548 \mathrm{~B} \times I$ Isingle $(\mathrm{FBS})_{\mathrm{ini}}$, the best fit was obtained for $\mathrm{A}=0.87$ and $\mathrm{B}=0.98$, demonstrating that 549 only $13 \%$ of the siRNA PMP22-SQ signal had disappeared. The same result has been 550 observed after incubation of siRNA PMP22-SQ NPs with a high concentration of bovine 551 serum albumin (BSA) $(200 \mu \mathrm{M}$, green pattern in Figure 5B). As with FBS, SANS patterns of 552 BSA alone (red pattern) or siRNA PMP22-SQ NPs alone (blue pattern) were also recorded. 553 Likewise, the scattering intensity was not much modified at small $q$. Thus, neither siRNA 554 PMP22-SQ NPs nor FBS or BSA were significantly affected by the presence of each other. 555 Therefore, it was concluded that siRNA PMP22-SQ NPs had a high colloidal stability in both 556 biological media.
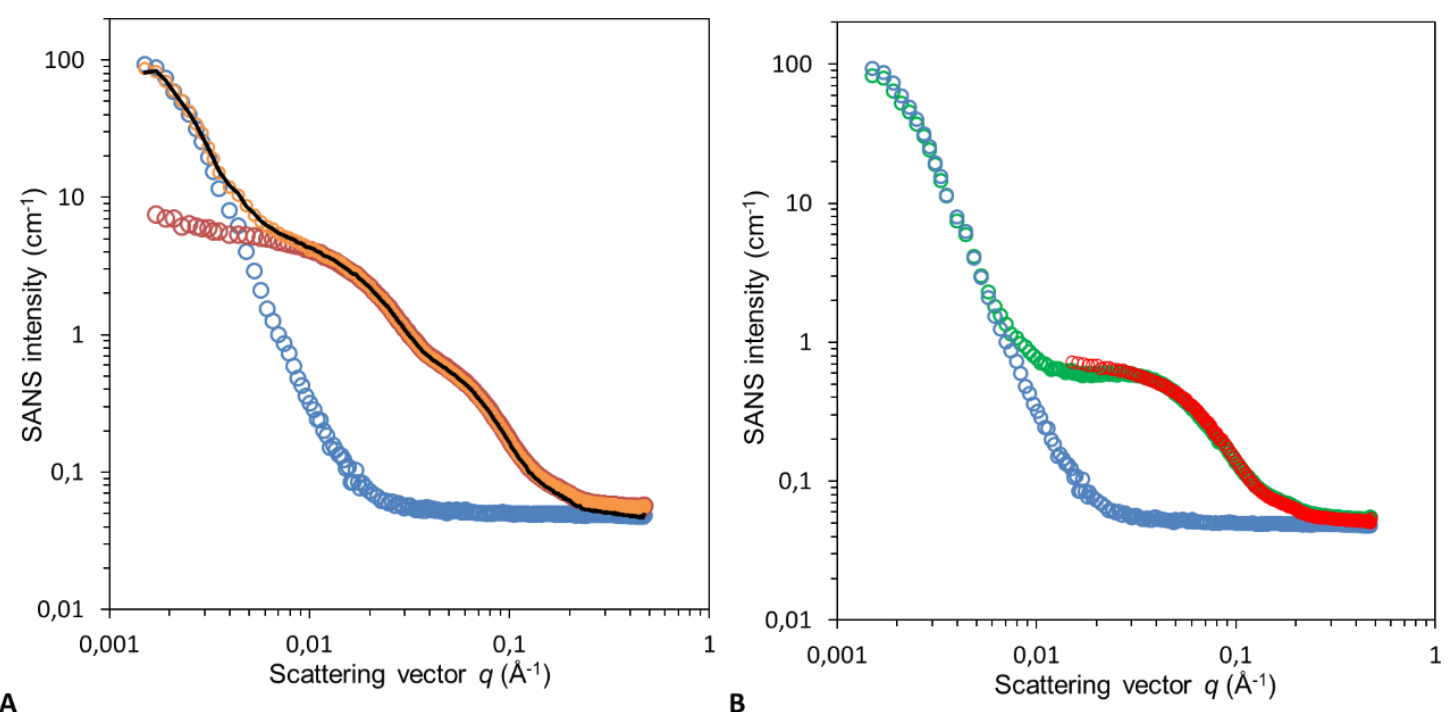
Figure 5: The siRNA PMP22-SQ NPs are stable in FBS and in the presence of high BSA concentration. A) SANS pattern of siRNA PMP22-SQ NPs/FBS mixture (yellow circles). Blue circles: $23 \mu \mathrm{M}$ [siRNA PMP22-SQ] in $\mathrm{D}_{2} \mathrm{O}$, red circles: FBS dialyzed against $\mathrm{D}_{2} \mathrm{O}$ and diluted by a factor of 2 to reach the same concentration as in siRNA PMP22-SQ NPs/FBS mixture. The smooth black curve corresponds to a linear combination of the FBS and siRNA PMP22-SQ NPs curves. B) SANS pattern of siRNA PMP22-SQ NPs/BSA mixture (green circles). Blue circles: $23 \mu \mathrm{M}$ [siRNA PMP22-SQ] in $\mathrm{D}_{2} \mathrm{O}$, red circles: $200 \mu \mathrm{M}$ [BSA] in $\mathrm{D}_{2} \mathrm{O}$.

The $\zeta$-potential increased progressively towards neutrality when siRNA PMP22-SQ NPs were incubated with $0.2 \%$ to $100 \%$ of FBS (Figure 6A). The $100 \%$ of FBS represent its $\zeta$-potential and is used as a control to discriminate the $\zeta$-potential of the nanoparticles from the undiluted FBS. These results highlighted the strong interaction occurring between some FBS components and siRNA PMP22-SQ NPs. Interaction with serum components was also confirmed by electrophoresis (Figure 6B). A progressive slowdown of migration reflected by a delay in their electrophoretic mobility was observed when siRNA PMP22-SQ NPs were incubated in FBS (respectively around $350 \mathrm{~kb}$ for NPs with FBS as against around $150 \mathrm{~kb}$ for NPs in $\mathrm{H}_{2} \mathrm{O}$ ). Interestingly, this phenomenon was observed at very low FBS concentration (2\%). Taken together, these results showed that FBS modified the surface charge and electrophoretic mobility of siRNA-SQ NPs.

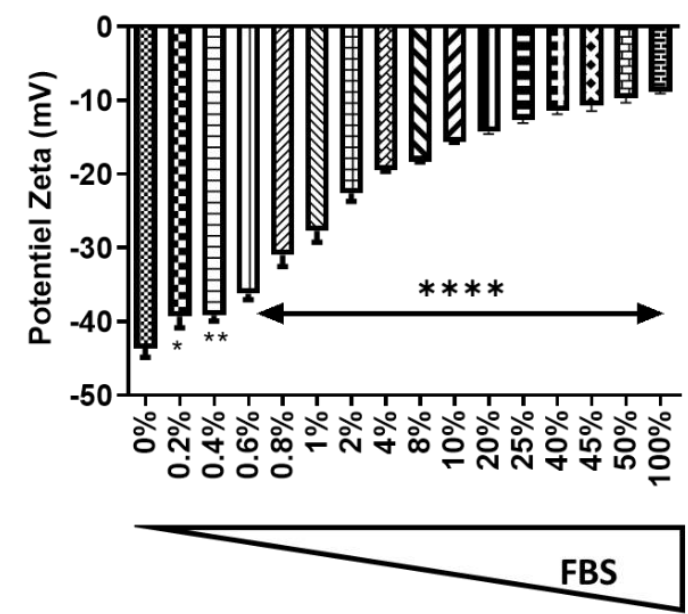

A

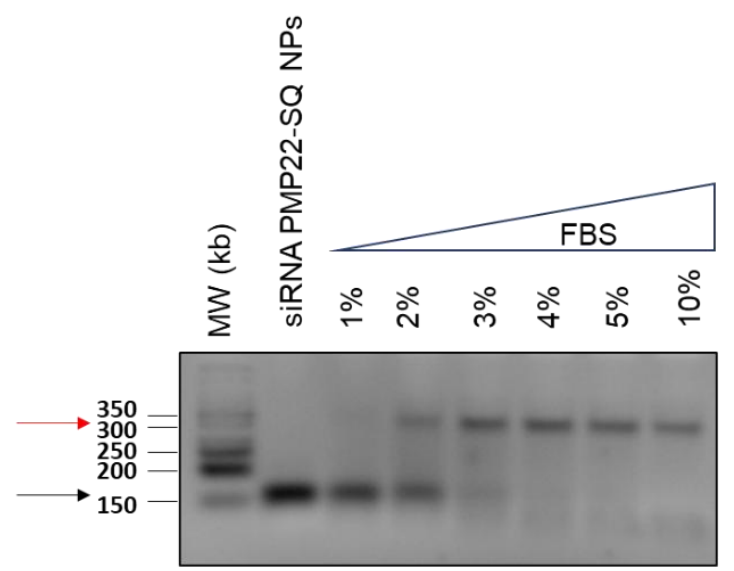

B
579

580

581

582

583

584

585
Figure 6: siRNA PMP22-SQ NPs interact with serum components. A) Measurements of the siRNA-PMP22-SQ NPs $\zeta$-potential in the presence of increasing concentrations of FBS (from pure water $(0 \% \mathrm{FBS})$ to pure FBS $(100 \%)$. $* \mathrm{p}<0.05, * * \mathrm{p}<0.001$ and $* * * * \mathrm{p}<0.00001$ by using One-way Anova followed by a Bonferroni test. B) The siRNA PMP22-SQ NPs were incubated with increased concentration of FBS and loaded onto 4\% agarose electrophoresis gel, then stained by Midori, a nucleotide maker. Migration slows down when FBS is added to 
the siRNA PMP22-SQ NPs (red arrow, wells 3 to 8), compared to siRNA PMP22-SQ NPs in water (black arrow, well 1).

\subsection{3. siRNA PMP22-SQ NPs interact with BSA}

Albumin is the main serum component, representing around 55\% of total protein content. BSA has intrinsic fluorescence, due to its amino acid, tryptophan, which can be recorded by fluorescence emission spectroscopy. Thus, the interaction between BSA and siRNA PMP22-SQ NPs was investigated by two fluorescence spectroscopic approach: SternVolmer quenching and steady-state fluorescence. First, by using the Stern-Volmer quenching experiment, we showed an interaction between siRNA PMP22-SQ NPs and BSA (Figure 7A). Indeed, when BSA was incubated with increasing siRNA PMP22-SQ concentrations, the intrinsic fluorescence of BSA was quenched, represented by a reduction of fluorescence emission compared to BSA alone at $5 \mu \mathrm{M}$ (red curve in Figure 7A). These results were determined according to the Stern-Volmer equation described in Supplementary information, equation $\mathbf{A}$. The value obtained for the quenching rate constant, $K_{\mathrm{q}}$, was higher than $10^{10} \mathrm{~mol} . \mathrm{L}^{-1} . \mathrm{s}^{-1}$, suggesting a static mechanism with the formation of a complex between BSA and siRNA PMP22-SQ NPs (Table 1). The equivalent of the affinity constant for the kinetic intermediate, the Stern-Volmer constant, was equal to $2.60 \pm 0.80 \times 10^{5} \mathrm{~L}^{\mathrm{mol}}{ }^{-1}$. All the obtained values are presented in Table 1.

Table 1: Numerical values based on linear fit of Stern-Volmer and Steady-state equations

\begin{tabular}{|c|c|c|c|}
\hline \multicolumn{2}{|l|}{ Constants } & Value & Unit \\
\hline Quenching rate constant & $\mathrm{K}_{\mathrm{q}}$ & $2.60 \pm 0.80$ & $\times 10^{13} \mathrm{~L} \cdot \mathrm{mol}^{-1} \cdot \mathrm{s}^{-1}$ \\
\hline $\begin{array}{l}\text { Stern-Volmer constant } \\
\text { (kinetic intermediate) }\end{array}$ & $\mathrm{K}_{\mathrm{sv}}$ & $2.60 \pm 0.80$ & $\times 10^{5} \mathrm{M}^{-1}$ \\
\hline $\begin{array}{l}\text { Overall affinity constant } \\
\text { (final thermodynamic complex) }\end{array}$ & $\mathrm{K}_{\mathrm{a}}$ & $2.34 \pm 0.59$ & $\mathrm{x} 10^{7} \mathrm{M}^{-1}$ \\
\hline
\end{tabular}

In the steady-state fluorescence approach, BSA was progressively titrated against siRNA PMP22-SQ NPs suspension, to a final molar ratio of 1 [siRNA PMP22-SQ]:1 [BSA].

608 In parallel, BSA was added to distilled water at the same concentration and the final spectra were compared. The presence of siRNA PMP22-SQ NPs led to a significant modification of

610 the BSA emission spectrum (red curve in Figure 7B) compared to that of BSA alone in water 611 (black curve in Figure 7B). Indeed, we observed a smaller fluorescence intensity under the 612 final experimental conditions and a shift in the maximum fluorescence intensity (from $351 \mathrm{~nm}$ 
613 for BSA alone to $349 \mathrm{~nm}$ for NPs-BSA), which indicated the formation of a complex between 614 the two species (i.e., siRNA PMP22-SQ NPs and BSA). The interaction mechanism could be 615 probed by several equations according to the species in solution that are dependent on $\mathrm{pH}$ and 616 temperature (Supplementary information, equation B). The linear least-squares regression 617 showed good correlation between siRNA PMP22-SQ NPs and BSA (Figure 7C). The 618 calculated overall apparent dissociation constant, $\mathrm{K}_{\mathrm{obs}}$, was equal to $4.95 \pm 1.38 \times 10^{-8} \mathrm{M}$ ( $\mathrm{n}=$ 6193 ; pH 7) with an overall affinity constant, $\mathrm{K}_{\mathrm{a}}$ of $2.34 \pm 0.59 \times 10^{7} \mathrm{M}^{-1}$.

620 Using both spectroscopic approaches, we showed the formation of a complex between 621 siRNA PMP22-SQ NPs and BSA. However, there was a disparity between the affinity 622 constants obtained. This could be explained by the experimental conditions, especially the 623 time (Figure 7D). Indeed, with Stern-Volmer quenching, a kinetic intermediate (BSA-NPs)' 624 was obtained with $\mathrm{K}_{\mathrm{a}}$ ' of $2.6 \times 10^{5} \mathrm{M}^{-1}$. The slow titration of the steady-state fluorescence led 625 to the final thermodynamic complex between BSA and NPs: BSA-NPs $\left(K_{a}=2.34 \pm 0.59 x\right.$ $62610^{7} \mathrm{M}^{-1}$ ). Therefore, the intermediate complex underwent a conformational change which 627 allowed to attempt its final thermodynamic state by increasing even more the stability of the 628 complex by a factor of 100 .
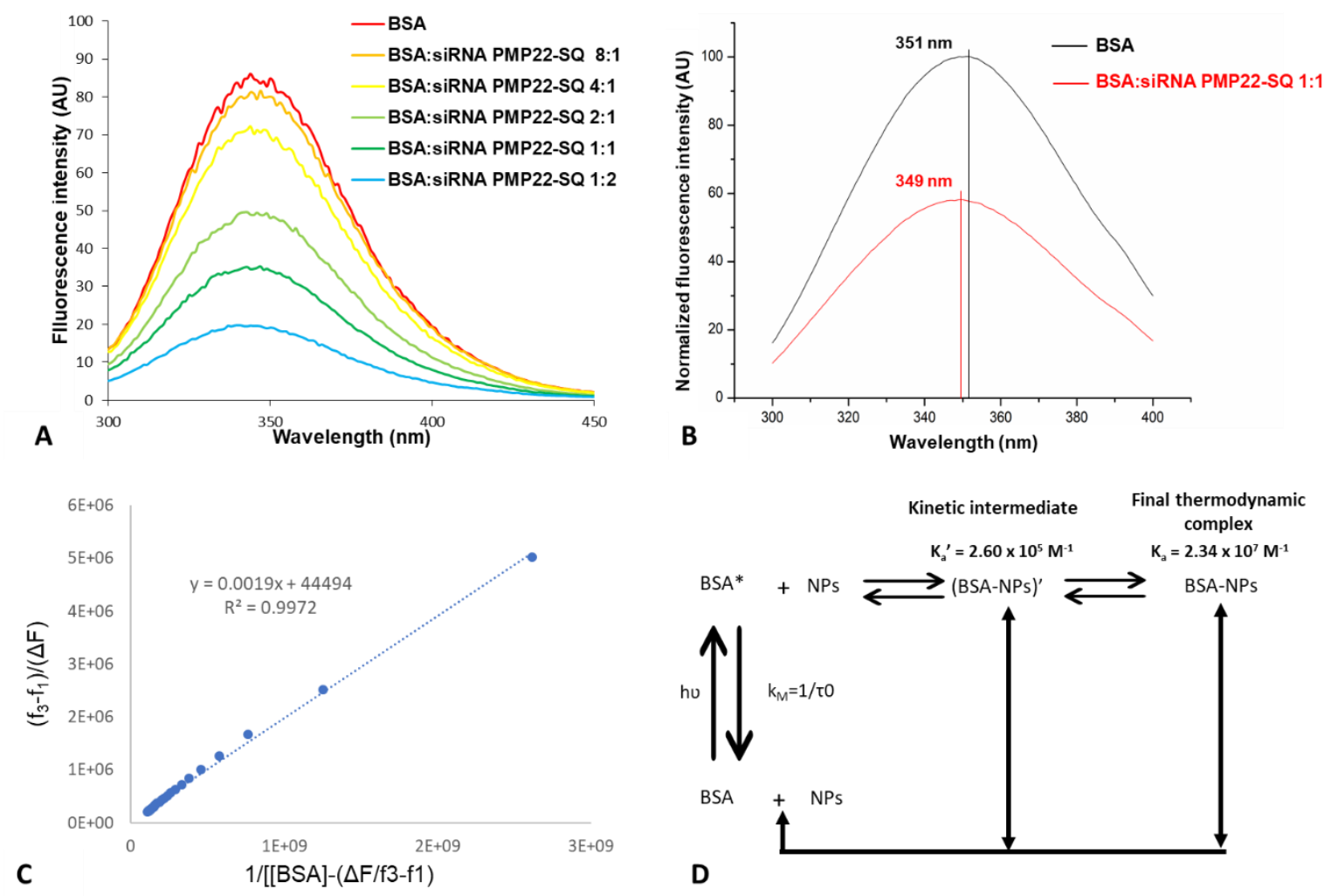

630 Figure 7: Spectroscopic analysis of siRNA PMP22-SQ NPs/BSA mixtures. A) Stern631 Volmer quenching of BSA in the presence of siRNA PMP22-SQ NPs. The fluorescence 632 intensity of BSA decreases progressively in the presence of siRNA PMP22-SQ NPs. B) 633 Steady-state experiment showing the emission fluorescence spectra at the end of the 
634 experiment, excitation wavelength $\left(\lambda_{\mathrm{ex}}=280 \mathrm{~nm}\right), \mathrm{pH} 7.4$ at $37^{\circ} \mathrm{C}$ : BSA $(5 \mu \mathrm{M}$, black curve $)$ 635 and BSA $(5 \mu \mathrm{M})+$ siRNA PMP22-SQ $(5 \mu \mathrm{M})$ in red. The maximal fluorescence intensity 636 decreases, and the emission wavelength shifts by $2 \mathrm{~nm}$ (from $351 \mathrm{~nm}$ to $349 \mathrm{~nm}$ ) when BSA 637 interacts with siRNA PMP22-SQ NPs. Fluorescence intensity has been normalized on BSA 638 maximum peak. C) Linear least-squares regression of the formula given in the 639 Supplementary information, equation $2\left(\mathrm{R}^{2}=0.9972\right)$. D) Graphical representation 640 showing the relationship between Stern-Volmer and steady-state approaches, showing the 641 affinity constants, $\mathrm{K}_{\mathrm{a}}$ ' and $\mathrm{K}_{\mathrm{a}}$

642 The interaction between BSA and siRNA PMP22-SQ NPs was further investigated by 643 circular dichroism spectroscopy (Figure 8A). Incubation of BSA with siRNA PMP22-SQ 644 NPs resulted in a decrease of $8 \%$ to $10 \%$ in the absolute signal intensity. Secondary structure 645 quantifications of BSA was performed by using BestSel software, showing a slight decrease 646 in the number of $\alpha$-helices, similarly to concentrated BSA (Figure S3). This interaction 647 between BSA and siRNA PMP22-SQ NPs was also tested by electrophoresis gel. Migration 648 of siRNA PMP22-SQ NPs was slowed down after incubation with increasing concentrations 649 of BSA (Figure 8B). At molar ratio [siRNA PMP22-SQ] 1: 4 [BSA], migration delay was 650 maximum and we estimated that around 10 BSA molecules surrounded 1 nanoparticle 651 (Supplementary information, equation C). Taken together, both results highlighted the 652 formation of a NPs-BSA complex. However, this complex was not able to modify the BSA 653 secondary structure.

654

655

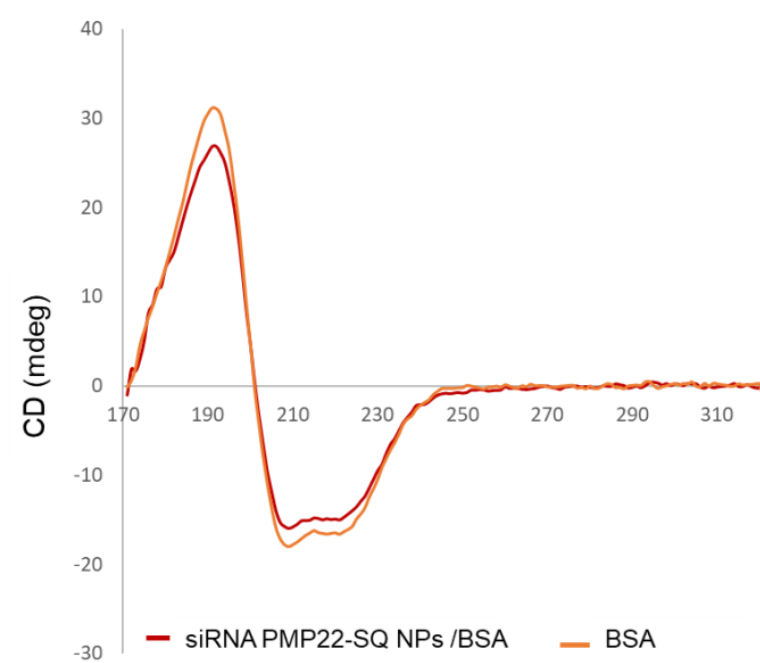

A

Figure 8: siRNA PMP22-SQ NPs interact with BSA. A) Circular dichroism spectrum of BSA alone (orange curve) and in the presence of siRNA PMP22-SQ NPs (red curve). In water, BSA spectra exhibits one positive peak at $192 \mathrm{~nm}$ and two negative bands at 210 and $224 \mathrm{~nm}$, both characteristic of the $\alpha$-helical structure. A decrease in the BSA signal in the presence of siRNA PMP22-SQ NPs is observed. B) Gel electrophoresis representing the migration of the siRNA PMP22-SQ NPs $(5 \mu \mathrm{M})$ incubated at $37^{\circ} \mathrm{C}$ with increasing $\mathrm{BSA}$ 
concentration loaded on 4\% agarose gel. Migration progressively slows down (red arrow, wells 4 to 8), compared to siRNA PMP22-SQ NPs in water (black arrow, well 1).

\subsection{4. siRNA-SQ NPs interact with LDL.}

Finally, we focused on the low-density lipoproteins (LDL), that carry $75 \%$ of blood cholesterol in humans. Incubation of siRNA PMP22-SQ NPs with increasing amounts of LDL showed a slowdown of the gel electrophoresis migration testifying an interaction between the two species (Figure 9A). In addition, $\zeta$-potential measurements revealed a significant progressive increase of the NPs surface charge with increasing LDL concentration (Figure 9B). These results were confirmed by the fluorescence spectrum of the LDL (Figure 9C), which weakened in the presence of the NPs and was accompanied by a 5-nm shift in the peak emission wavelength. This clearly confirmed the formation of a siRNA PMP22-SQ NPs LDL complex.

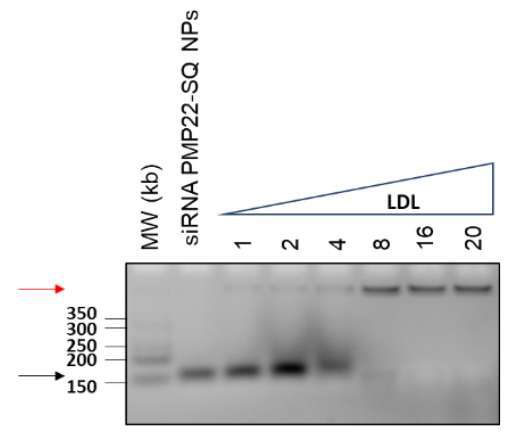

A

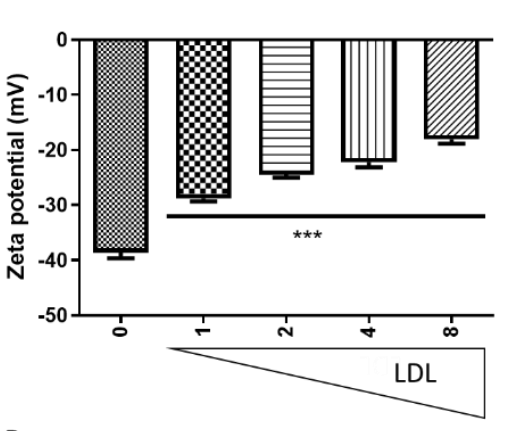

B

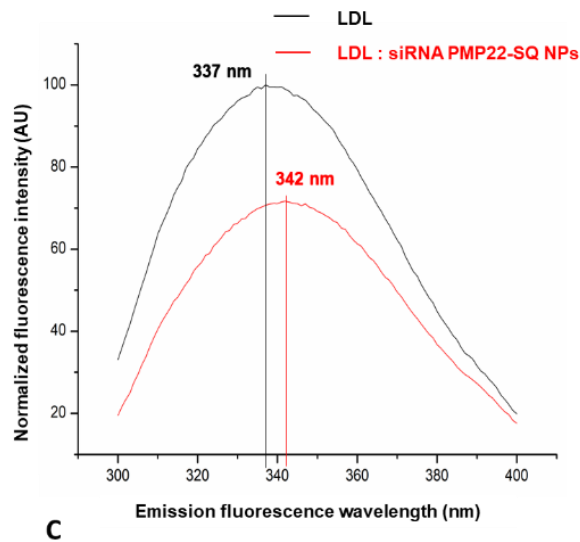

C

Figure 9: siRNA-SQ NPs interact with LDLs. A) Gel electrophoresis showing the migration of siRNA PMP22-SQ NPs incubated at $37^{\circ} \mathrm{C}$ with increasing LDL concentration loaded on $4 \%$ agarose gel. Migration progressively slows down (red arrow, wells 5 to 7), compared to siRNA PMP22-SQ NPs in water (black arrow, well 1). B) $\zeta$-potential measurement after incubation with growing concentrations of LDL. $* * * p<0.0001$ vs siRNA PMP22-SQ NPs (One-way Anova followed by a Bonferroni test). C) Emitted fluorescence spectrum of LDL in the absence (black curve) or presence of siRNA-SQ NPs (red curve). The maximal fluorescence intensity decreases and there is a $5 \mathrm{~nm}$ shift of emission peak (from 337 $\mathrm{nm}$ to $342 \mathrm{~nm}$ ) when LDL interacts with siRNA PMP22-SQ NPs. Fluorescence intensity has been normalized to LDL peak maximum.

3.2.5. siRNA PMP22-SQ NPs interact via the squalene moiety with serum components

NPs made from small molecules linked to squalene, including nucleoside analogs, interact with serum components via the SQ moiety (Sobot, Mura et al. 2017, Sobot, Mura et al. 2017, Yesylevskyy, Ramseyer et al. 2018). To confirm that similar interaction could occur 
with siRNA PMP22-SQ NPs and FBS or its components (i.e., BSA and LDL), naked siRNA PMP22 (i.e. not conjugated with SQ) was incubated with increasing amounts of whole serum, BSA or LDL, before electrophoresis were performed. Contrary to siRNA PMP22-SQ NPs, migration of serum and serum components did not slow down (Figure 10). This confirmed that the interaction of nanoparticles with serum components occurred well via the squalene moiety and not via the oligonucleotide moiety.

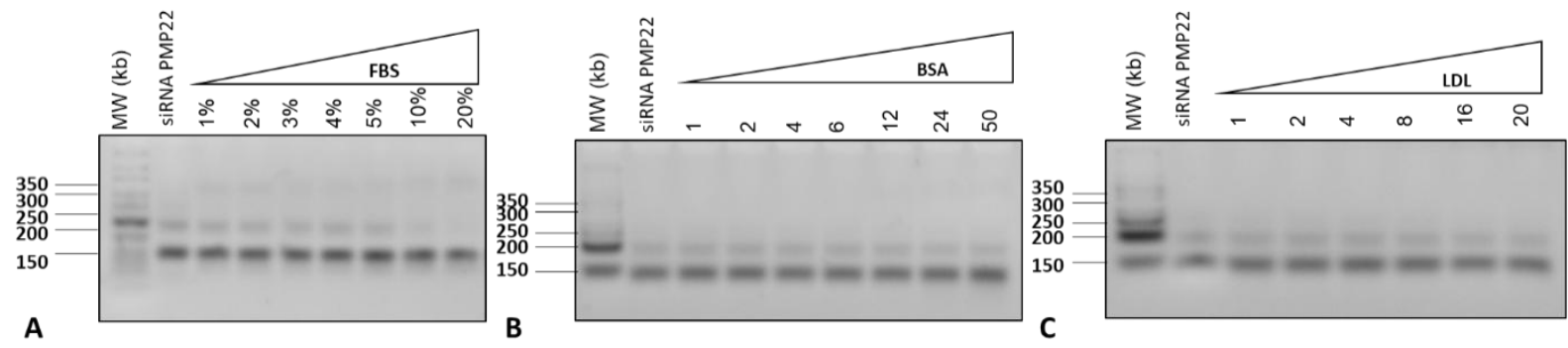

Figure 10: Naked siRNA PMP22 do not interact with total serum, BSA and LDL. 4\% non-denaturated agarose electrophoresis were performed after incubation of naked siRNA PMP22 with increasing amounts of FBS (A), BSA (B, molar ratio) and LDL ( $\mathbf{C}$, mass ratio). No slowdown of migration was observed.

\section{Discussion}

Conjugation of siRNA to squalene by Cu-free click chemistry, has been previously demonstrated to protect the double-strand oligonucleotide from degradation and to treat different experimental diseases such as cancer with fusion oncogene or peripheral neuropathy (Massaad-Massade, Boutary et al. 2018, Boutary, Caillaud et al. 2021). To be injectable and efficient in vivo, squalenoyl siRNAs needed to be formulated as nanoparticles. However, due to the different molecular weight of the two bioconjugate moieties (i.e., 13,000 Da for siRNA / 494 Da for SQ forming a ratio of 26/1 between hydrophilic siRNA and lipophilic squalene) and because the siRNA-SQ bioconjugates were not soluble in organic solvents but totally soluble in water, an inverse nanoprecipitation protocol has been optimized by adding the aqueous phase to the organic one, before solvent evaporation to obtain the final siRNASQ NPs suspension (Bilati, Allémann et al. 2005).

The size and size distribution of the nanoparticles are important parameters for drug delivery systems. Using DLS, a rapid method for determination of nanoparticles size, we found that the diameter of squalenoyl siRNA nanoparticles were nucleoside sequence independent. Indeed, both siRNA PMP22-SQ and RET/PTC1-SQ nanoparticles displayed similar dimension, around $200 \mathrm{~nm}$ with a negative charge less than $-30 \mathrm{mV}$; they were stable for a month at $4{ }^{\circ} \mathrm{C}$ (Massaad-Massade, Boutary et al. 2018, Boutary, Caillaud et al. 2021). As no significant difference was found concerning their physicochemical parameters, it was 
decided to use the siRNA PMP22-SQ NPs for all further experiments. This resolution was also supported by our recent findings concerning the therapeutic efficiency of siRNA PMP22SQ NPs as a targeted therapy for CMT1A neuropathy (Boutary, Caillaud et al. 2021).

Then, the siRNA PM22-SQ NPs were observed by cryo-TEM. Spherical nanoobjects of around $130 \mathrm{~nm}$ in size with no specific internal structure were observed similar to our previous observation on siRNA TMPRSS2-ERG-SQ NPs, which confirmed once again that the size was independent of the nucleoside sequence (Massaad-Massade, Boutary et al. 2018). Of note, cryoTEM is more specific than DLS, since it provides additional information on shape and morphology. The smaller nanoparticles size observed by cryoTEM was explained by the fact that laser light scattering measured the hydrodynamic size when cryoTEM allowed to visualize the nanoparticle core only. Small-angle neutron scattering (SANS) confirmed the observed size and shape of the siRNA PMP22-SQ nanoparticles. Altogether DLS, cryo-TEM and SANS analysis represented complementary approaches, giving an ensemble picture of the siRNA PMP22-SQ NPs.

To describe more precisely the internal composition and the siRNA organization inside of the NPs, SAXS analysis have been performed, which depicted that the siRNA PMP22-SQ bioconjugates self-organized in water into small core/shell domains of 11-nm size with the lipidic moiety exposed to the outside and the siRNA located in the core, without modification of the $\alpha$-helix structure of the nucleic acid. We assume that hydrophobic interactions are the main driving force leading to the self-assembly of the nanoparticle into a 130-nm size spherical random structure with some hydrophilic siRNA moieties being exposed onto the nanoparticle surface for solubility reasons. To our knowledge, this unique random supramolecular structure, composed of typical 11-nm size spheres with internal electronic density heterogeneities, has only been observed with siRNA PMP22-SQ NPs. It confirms the diversity of the squalenoyl nanostructures and the flexibility of the hydrophobic squalene chain which allows to self-assemble into nanoparticles, even when a hydrophilic macromolecule of much higher molecular weight was attached to the SQ.

We then assessed the interaction of siRNA PMP22-SQ NPs with blood components, specifically albumin and lipoproteins. Indeed, once administered intravenously, siRNA-SQ NPs encounter different blood components that can adsorb to the NPs surface, either stabilizing or destabilizing their structure and this may dramatically impact their in vivo fate in terms of biodistribution, pharmacokinetics, toxicity and therapeutic efficiency (Aggarwal, 756 Hall et al. 2009, Lesniak, Fenaroli et al. 2012, Monopoli, Aberg et al. 2012, Gao and He 2014, 
It was observed that siRNA PMP22-SQ nanoparticles interacted hydrophobically, via the squalene moiety, with serum components, especially BSA and LDL. Interestingly, it has been reported that BSA may display some dysopsonin-like activity (Ogawara, Furumoto et al. 2004). These interactions with serum components can undergo conformational changes on the NPs surface which could also affect their biological fates (Gunawan, Lim et al. 2014). This hypothesis was assessed by SANS experiments performed in FBS and in presence of high BSA concentrations. No significant modifications in the SANS pattern of the nanoparticles could be observed. This proved that the siRNA PMP22-SQ NPs kept high colloidal stability in biological media, contrarily to previous studies performed with small chemical molecules conjugated to squalene showing a destabilization of the squalenoyl NPs in the presence of blood components, with an insertion of these bioconjugates, as molecular entities, into the LDL and BSA fractions (Ambike, Rosilio et al. 2011, Sobot, Mura et al. 2017, Yesylevskyy, Ramseyer et al. 2018, Gobeaux, Bizeau et al. 2020). Thus, the present study clearly suggests that the biological behavior of squalene-based nanoparticles may depend on the nature of the associated biologically active molecule. Such different behavior of squalenoyl NPs may be explained by: i) the respective molecular weight of the linked drug versus the squalene (i.e., a ratio of 1 or $2 v s 26$ with siRNA-SQ), ii) the different physicochemical parameters (siRNASQ is highly negatively charged) and, iii) the supramolecular organization.

Since LDL and HDL are the main transporters of cholesterol, an important lipid for tumor cell proliferation (Chaudhary, Bower et al. 2019) and myelin synthesis (Zhou, Bazick et al. 2019), we hypothesize that the interaction of LDL with siRNA PMP22-SQ NPs may trigger a natural nanoparticles functionalization for targeting these pathological tissues. Additionally, such specific biodistribution may be amplified by the inflammation of the pathological tissues, resulting in histological modifications such as defective endothelial cells with wide fenestrations in cancer tissues (Tatiparti, Sau et al. 2017, Hoogenboezem and Duvall 2018) and edema with lymphocyte infiltration in neurodegenerative diseases (Weis, Claeys et al. 2017). Especially, albumin accumulate preferentially in these type of tissues (Hoogenboezem and Duvall 2018). Thus, siRNA-SQ NPs are expected to extravasate through damaged tissues, as supported by our previous studies for cancers and a disease of the peripheral nervous system (CMT1A), where in both pathologies a significant inhibition of the targeted gene was observed in the tissues concerned (Massaad-Massade, Boutary et al. 2018, Boutary, Caillaud et al. 2021).

\section{Conclusions}


791 The present study has provided an important contribution to better understand the 792 physico-chemical structures of siRNA squalene-based nanoparticles, as well as their 793 interactions with some biological components encountered after intravenous administration. 794 The high specificity of siRNA to decrease or normalize gene expression offers new outlooks 795 to cure pathologies with gene expression dysregulation such as fusion oncogene cancer or 796 hereditary monogenic peripheral neuropathy. In this area, siRNA-SQ NPs are promising new 797 potential drug candidates with wide therapeutic applications thanks to their high colloidal 798 stability.

799 
M.C. designed and performed the experiments and wrote the manuscript, F.G. contributed and performed the experiments of SAXS and SANS studies, M.H. developed the

803 Fluorescence quenching experiments, S.B. contributed to the synthesis and characterization of 804 the siRNA-SQ NPs, P.G. contributed to study design and discussion, D.D developed the azido-squalene synthesis, P.C. contribute on the discussion of the results and to the writing of the manuscript, F.W. and I.G. performed with MC and FG the SRCD experiments, F.T. contributed and performed the experiments of SAXS and SANS studies and, L.M. is the PI of the study, she supervised, designed the study and contributed to the writing of the manuscript.

\section{Declaration of Competing Interest}

The authors declare that they have no known competing financial interests or personal relationships that could have appeared to influence the work reported in this paper.

\section{Acknowledgment}

This paper is dedicated to Ms. Isabelle Grillo $†$ (Institut Laue Langevin, 71 avenue des Martyrs, B.P. 156, 38042 Grenoble Cedex 9, France) who passed away. Ms Grillo performed the SRCD experiments.

We thank for Julien Loisel Duwattez and Alexandre Rodrigues for their technical assistance, David Carrière for SAXS-WAXS measurements performed on the SWING beamline (SOLEIL Synchrotron) during the BAG 20181790 and 20201118. SRCD experiments were performed on the DISCO Beamline at the SOLEIL Synchrotron (Saint Aubin, France). This work benefited from the use of the SasView application (http://www.sasview.org), originally developed under NSF award DMR-0520547. SasView contains code developed with funding from the European Union's Horizon 2020 research and innovation program under the SINE2020 project, grant agreement no. 654000.

The authors also thank Ghislaine Frebourg for Cryo-TEM observations (Electronic Microscopy Platform, IBPS/Institut de Biologie Paris-Seine, Université P. et M. Curie, Paris, France) and John S. Lomas (Interfaces, Traitements, Organisation et DYnamique des Systèmes (ITODYS), UFR de Chimie 75013 Paris, France) for the English editing. Marie Caillaud is grateful for the financial support of the Ile-de-France region within the framework 
832 Aggarwal, P., J. B. Hall, C. B. McLeland, M. A. Dobrovolskaia and S. E. McNeil (2009). "Nanoparticle

833 interaction with plasma proteins as it relates to particle biodistribution, biocompatibility and

834 therapeutic efficacy." Adv Drug Deliv Rev 61(6): 428-437.

835 Ali, H. M., A. Maksimenko, G. Urbinati, H. Chapuis, M. Raouane, D. Desmaele, H. Yasuhiro, H.

836 Harashima, P. Couvreur and L. Massaad-Massade (2014). "Effects of silencing the RET/PTC1

837 oncogene in papillary thyroid carcinoma by siRNA-squalene nanoparticles with and without fusogenic companion GALA-cholesterol." Thyroid 24(2): 327-338.

838 Ali, H. M., G. Urbinati, H. Chapuis, D. Desmaele, J. R. Bertrand, P. Couvreur and L. Massaad-Massade

840 (2014). "Effects of siRNA on RET/PTC3 junction oncogene in papillary thyroid carcinoma: from molecular and cellular studies to preclinical investigations." PLoS One 9(4): e95964. Ali, H. M., G. Urbinati, M. Raouane and L. Massaad-Massade (2012). "Significance and applications of nanoparticles in siRNA delivery for cancer therapy." Expert Rev Clin Pharmacol 5(4): 403-412. Ambike, A., V. Rosilio, B. Stella, S. Lepêtre-Mouelhi and P. Couvreur (2011). "Interaction of selfassembled squalenoyl gemcitabine nanoparticles with phospholipid-cholesterol monolayers mimicking a biomembrane." Langmuir 27(8): 4891-4899. Bertrand, J. R., C. Lucas, N. M. Pham, C. Durieu, P. Couvreur, C. P. Malvy and D. Desmaele (2015). "Turning Squalene into Cationic Lipid Allows a Delivery of siRNA in Cultured Cells." Nucleic Acid Ther 25(3): 121-129. Bilati, U., E. Allémann and E. Doelker (2005). "Development of a nanoprecipitation method intended for the entrapment of hydrophilic drugs into nanoparticles." Eur J Pharm Sci 24(1): 67-75. Boutary, S., M. Caillaud, M. El Madani, J.-M. Vallat, J. Loisel-Duwattez, A. Rouyer, L. Richard, C. Gracia, G. Urbinati, D. Desmaële, A. Echaniz-Laguna, D. Adams, P. Couvreur, M. Schumacher, C. Massaad and L. Massaad-Massade (2021). "Squalenoyl siRNA PMP22 nanoparticles are effective in treating mouse models of Charcot-Marie-Tooth disease type 1 A." Communications Biology 4(1): 317. Boutary, S., M. Caillaud, M. El Madani, J. M. Vallat, J. Loisel-Duwattez, A. Rouyer, L. Richard, C. Gracia, G. Urbinati, D. Desmaële, A. Echaniz-Laguna, D. Adams, P. Couvreur, M. Schumacher, C. Massaad and L. Massaad-Massade (2021). "Squalenoyl siRNA PMP22 nanoparticles are effective in treating mouse models of Charcot-Marie-Tooth disease type 1 A." Commun Biol 4(1): 317.

Caillaud, M., M. El Madani and L. Massaad-Massade (2020). "Small interfering RNA from the lab discovery to patients' recovery." J Control Release 321: 616-628.

Chaudhary, J., J. Bower and I. R. Corbin (2019). "Lipoprotein Drug Delivery Vehicles for Cancer: Rationale and Reason." Int J Mol Sci 20(24). Couvreur, P., L. H. Reddy, S. Mangenot, J. H. Poupaert, D. Desmaele, S. Lepetre-Mouelhi, B. Pili, C. Bourgaux, H. Amenitsch and M. Ollivon (2008). "Discovery of new hexagonal supramolecular nanostructures formed by squalenoylation of an anticancer nucleoside analogue." Small 4(2): 247253.

Couvreur, P., B. Stella, L. H. Reddy, H. Hillaireau, C. Dubernet, D. Desmaële, S. Lepêtre-Mouelhi, F. Rocco, N. Dereuddre-Bosquet, P. Clayette, V. Rosilio, V. Marsaud, J.-M. Renoir and L. Cattel (2006). "Squalenoyl Nanomedicines as Potential Therapeutics." Nano Letters 6(11): 2544-2548.

Desmaele, D., R. Gref and P. Couvreur (2012). "Squalenoylation: a generic platform for nanoparticular drug delivery." J Control Release 161(2): 609-618.

Dormont, F., M. Rouquette, C. Mahatsekake, F. Gobeaux, A. Peramo, R. Brusini, S. Calet, F. Testard, S. Lepetre-Mouelhi, D. Desmaële, M. Varna and P. Couvreur (2019). "Translation of nanomedicines from lab to industrial scale synthesis: The case of squalene-adenosine nanoparticles." J Control Release 307: 302-314.

Elbashir, S. M. (2001). "RNA interference is mediated by 21- and 22-nucleotide RNAs." Genes \& Development 15(2): 188-200. 
Elbashir, S. M., J. Martinez, A. Patkaniowska, W. Lendeckel and T. Tuschl (2001). "Functional anatomy of siRNAs for mediating efficient RNAi in Drosophila melanogaster embryo lysate." Embo j 20(23):

\section{6877-6888.}

882 Fire, A., S. Xu, M. K. Montgomery, S. A. Kostas, S. E. Driver and C. C. Mello (1998). "Potent and 883 specific genetic interference by double-stranded RNA in Caenorhabditis elegans." Nature 391(6669):

\section{806-811.}

885 Gao, H. and Q. He (2014). "The interaction of nanoparticles with plasma proteins and the consequent 886 influence on nanoparticles behavior." Expert Opin Drug Deliv 11(3): 409-420. Gobeaux, F., J. Bizeau, F. Samson, L. Marichal, I. Grillo, F. Wien, S. O. Yesylevsky, C. Ramseyer, M. Rouquette, S. Lepêtre-Mouelhi, D. Desmaële, P. Couvreur, P. Guenoun, J. P. Renault and F. Testard (2020). "Albumin-driven disassembly of lipidic nanoparticles: the specific case of the squaleneadenosine nanodrug." Nanoscale 12(4): 2793-2809.

Guinier, A., G. Fournet and K. L. Yudowitch (1955). "Small-angle scattering of X-rays." Gunawan, C., M. Lim, C. P. Marquis and R. Amal (2014). "Nanoparticle-protein corona complexes govern the biological fates and functions of nanoparticles." J Mater Chem B 2 (15): 2060-2083. Hoogenboezem, E. N. and C. L. Duvall (2018). "Harnessing albumin as a carrier for cancer therapies." Adv Drug Deliv Rev 130: 73-89.

Kanasty, R., J. R. Dorkin, A. Vegas and D. Anderson (2013). "Delivery materials for siRNA therapeutics." Nature Materials 12: 967.

Kang, M., H. Kim and C. Leal (2016). "Self-organization of Nucleic Acids in Lipid Constructs." Curr Opin Colloid Interface Sci 26: 58-65.

Kratz, F. (2008). "Albumin as a drug carrier: design of prodrugs, drug conjugates and nanoparticles." 」 Control Release 132(3): 171-183.

Lamichhane, S. and S. Lee (2020). "Albumin nanoscience: homing nanotechnology enabling targeted drug delivery and therapy." Arch Pharm Res 43(1): 118-133.

Lepeltier, E., C. Bourgaux and P. Couvreur (2014). "Nanoprecipitation and the "Ouzo effect": Application to drug delivery devices." Adv Drug Deliv Rev 71: 86-97.

Lepeltier, E., C. Bourgaux, A. Maksimenko, F. Meneau, V. Rosilio, E. Sliwinski, F. Zouhiri, D. Desmaële and P. Couvreur (2014). "Self-assembly of polyisoprenoyl gemcitabine conjugates: influence of supramolecular organization on their biological activity." Langmuir 30(22): 6348-6357.

Lesniak, A., F. Fenaroli, M. P. Monopoli, C. Åberg, K. A. Dawson and A. Salvati (2012). "Effects of the presence or absence of a protein corona on silica nanoparticle uptake and impact on cells." ACS Nano 6(7): 5845-5857.

Mariam, J., S. Sivakami and P. M. Dongre (2016). "Albumin corona on nanoparticles - a strategic approach in drug delivery." Drug Deliv 23(8): 2668-2676.

Marichal, L., J. Degrouard, A. Gatin, N. Raffray, J. C. Aude, Y. Boulard, S. Combet, F. Cousin, S. Hourdez, J. Mary, J. P. Renault and S. Pin (2020). "From Protein Corona to Colloidal Self-Assembly: The Importance of Protein Size in Protein-Nanoparticle Interactions." Langmuir 36(28): 8218-8230. Marichal, L., G. Giraudon-Colas, F. Cousin, A. Thill, J. Labarre, Y. Boulard, J. C. Aude, S. Pin and J. P. Renault (2019). "Protein-Nanoparticle Interactions: What Are the Protein-Corona Thickness and Organization?" Langmuir 35(33): 10831-10837.

Massaad-Massade, L., S. Boutary, M. Caillaud, C. Gracia, B. Parola, S. B. Gnaouiya, B. Stella, S. Arpicco, E. Buchy, D. Desmaele, P. Couvreur and G. Urbinati (2018). "New Formulation for the Delivery of Oligonucleotides Using "Clickable" siRNA-Polyisoprenoid-Conjugated Nanoparticles: Application to Cancers Harboring Fusion Oncogenes." Bioconjug Chem 29(6): 1961-1972.

Miles, A. J. and B. A. Wallace (2018). "CDtoolX, a downloadable software package for processing and analyses of circular dichroism spectroscopic data." Protein Science 27(9): 1717-1722.

Monopoli, M. P., C. Aberg, A. Salvati and K. A. Dawson (2012). "Biomolecular coronas provide the biological identity of nanosized materials." Nat Nanotechnol 7(12): 779-786.

Ogawara, K., K. Furumoto, S. Nagayama, K. Minato, K. Higaki, T. Kai and T. Kimura (2004). "Precoating with serum albumin reduces receptor-mediated hepatic disposition of polystyrene nanosphere: implications for rational design of nanoparticles." J Control Release 100(3): 451-455. 
Ozpolat, B., A. K. Sood and G. Lopez-Berestein (2014). "Liposomal siRNA nanocarriers for cancer therapy." Adv Drug Deliv Rev 66: 110-116.

Raouane, M., D. Desmaele, M. Gilbert-Sirieix, C. Gueutin, F. Zouhiri, C. Bourgaux, E. Lepeltier, R. Gref, R. Ben Salah, G. Clayman, L. Massaad-Massade and P. Couvreur (2011). "Synthesis, characterization, and in vivo delivery of siRNA-squalene nanoparticles targeting fusion oncogene in papillary thyroid carcinoma." J Med Chem 54(12): 4067-4076. Richard, D., M. Ferrand and G. Kearley (1996). "Analysis and visualisation of neutron-scattering data." Journal of Neutron Research 4(1-4): 33-39. Ristori, S., L. Ciani, G. Candiani, C. Battistini, A. Frati, I. Grillo and M. In (2012). "Complexing a small interfering RNA with divalent cationic surfactants." Soft Matter 8(3): 749-756. Rouquette, M., K. Ser-Le Roux, M. Polrot, C. Bourgaux, J. P. Michel, F. Testard, F. Gobeaux and S. Lepetre-Mouelhi (2019). "Towards a clinical application of freeze-dried squalene-based nanomedicines." J Drug Target 27(5-6): 699-708.

Saha, D., F. Testard, I. Grillo, F. Zouhiri, D. Desmaele, A. Radulescu, S. Desert, A. Brulet, P. Couvreur and O. Spalla (2015). "The role of solvent swelling in the self-assembly of squalene based nanomedicines." Soft Matter 11(21): 4173-4179.

Schumaker, V. N. and G. H. Adams (1969). "Circulating lipoproteins." Annu Rev Biochem 38: 113-136. Sleep, D. (2015). "Albumin and its application in drug delivery." Expert Opin Drug Deliv 12(5): 793 812.

Sobot, D., S. Mura, M. Rouquette, B. Vukosavljevic, F. Cayre, E. Buchy, G. Pieters, S. Garcia-Argote, M. Windbergs, D. Desmaele and P. Couvreur (2017). "Circulating Lipoproteins: A Trojan Horse Guiding Squalenoylated Drugs to LDL-Accumulating Cancer Cells." Mol Ther 25(7): 1596-1605. Sobot, D., S. Mura, S. O. Yesylevskyy, L. Dalbin, F. Cayre, G. Bort, J. Mougin, D. Desmaele, S. LepetreMouelhi, G. Pieters, B. Andreiuk, A. S. Klymchenko, J. L. Paul, C. Ramseyer and P. Couvreur (2017). "Conjugation of squalene to gemcitabine as unique approach exploiting endogenous lipoproteins for drug delivery." Nat Commun 8: 15678.

Tatiparti, K., S. Sau, S. K. Kashaw and A. K. Iyer (2017). "siRNA Delivery Strategies: A Comprehensive Review of Recent Developments." Nanomaterials (Basel) 7(4).

Thompson, J. D., D. J. Kornbrust, J. W. Foy, E. C. Solano, D. J. Schneider, E. Feinstein, B. A. Molitoris and S. Erlich (2012). "Toxicological and pharmacokinetic properties of chemically modified siRNAs targeting p53 RNA following intravenous administration." Nucleic Acid Ther 22(4): 255-264.

Tuschl, T. (2002). Expanding small RNA interference. Nat Biotechnol. United States. 20: 446-448. Urbinati, G., H. M. Ali, Q. Rousseau, H. Chapuis, D. Desmaele, P. Couvreur and L. Massaad-Massade (2015). "Antineoplastic Effects of siRNA against TMPRSS2-ERG Junction Oncogene in Prostate Cancer." PLoS One 10(5): e0125277.

Urbinati, G., H. M. Ali, Q. Rousseau, H. Chapuis, D. Desmaele, P. Couvreur and L. Massaad-Massade (2015). "Antineoplastic Effects of siRNA against TMPRSS2-ERG Junction Oncogene in Prostate Cancer." Plos One 10(5): 23.

Urbinati, G., I. de Waziers, M. Slamic, T. Foussigniere, H. M. Ali, D. Desmaele, P. Couvreur and L. Massaad-Massade (2016). "Knocking Down TMPRSS2-ERG Fusion Oncogene by siRNA Could be an Alternative Treatment to Flutamide." Mol Ther Nucleic Acids 5: e301. Urbinati, G., I. de Waziers, M. Slamic, T. Foussigniere, H. M. Ali, D. Desmaele, P. Couvreur and L. Massaad-Massade (2016). "Knocking Down TMPRSS2-ERG Fusion Oncogene by siRNA Could be an Alternative Treatment to Flutamide." Molecular Therapy-Nucleic Acids 5: 12. Urbinati, G., A. M. Hafiz and L. Massaad-Massade (2013). "New pharmacological approach by siRNA against junction oncogenes for thyroid and prostate cancers." International Journal of Molecular Medicine 32: S37-S37.

Wasan, K. M., D. R. Brocks, S. D. Lee, K. Sachs-Barrable and S. J. Thornton (2008). "Impact of lipoproteins on the biological activity and disposition of hydrophobic drugs: implications for drug discovery." Nat Rev Drug Discov 7(1): 84-99.

Weis, J., K. G. Claeys, A. Roos, H. Azzedine, I. Katona, J. M. Schröder and J. Senderek (2017). "Towards a functional pathology of hereditary neuropathies." Acta Neuropathol 133(4): 493-515. 
983 Whitehead, K. A., R. Langer and D. G. Anderson (2009). "Knocking down barriers: advances in siRNA 984 delivery." Nat Rev Drug Discov 8(2): 129-138.

985 Wolfrum, C., S. Shi, K. N. Jayaprakash, M. Jayaraman, G. Wang, R. K. Pandey, K. G. Rajeev, T.

986 Nakayama, K. Charrise, E. M. Ndungo, T. Zimmermann, V. Koteliansky, M. Manoharan and M. Stoffel

987 (2007). "Mechanisms and optimization of in vivo delivery of lipophilic siRNAs." Nat Biotechnol 25(10):

$988 \quad 1149-1157$.

989 Yesylevskyy, S. O., C. Ramseyer, M. Savenko, S. Mura and P. Couvreur (2018). "Low-Density

990 Lipoproteins and Human Serum Albumin as Carriers of Squalenoylated Drugs: Insights from

991 Molecular Simulations." Mol Pharm 15(2): 585-591.

992 Zhou, Y., H. Bazick, J. R. Miles, A. I. Fethiere, M. O. Al Salihi, S. Fazio, H. Tavori and L. Notterpek

993 (2019). "A neutral lipid-enriched diet improves myelination and alleviates peripheral nerve pathology

994 in neuropathic mice." Experimental neurology 321: 113031.

995

996 


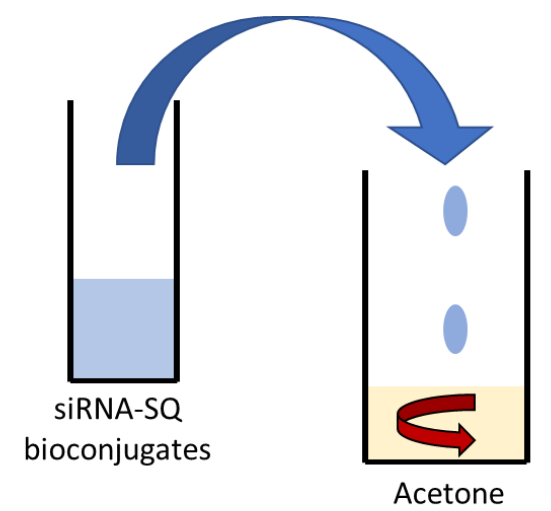

Dropwise inverse nanoprecipitation Ratio V/V 1 (acetone) : 2 (siRNA-SQ in water)

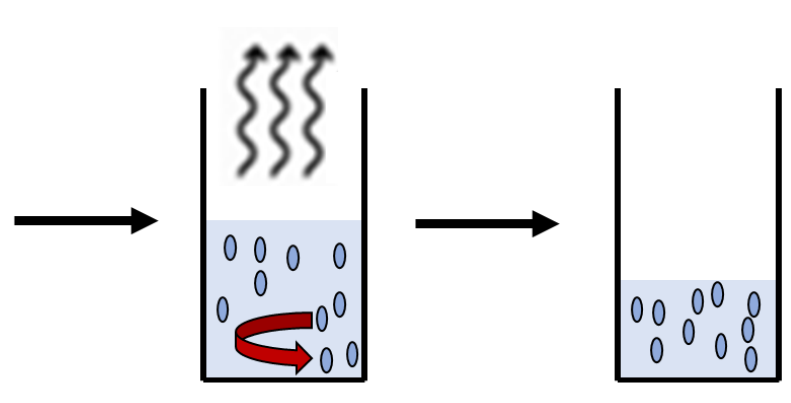

Acetone evaporation under $\mathrm{N}_{2}$ siRNA-SQ NPs solution flux (30min)
Figure S1: Sketch of siRNA-SQ nanoparticles preparation. The siRNA-SQ bioconjugates were obtained after $\mathrm{Cu}$-free click chemistry and annealing to the antisense strand. Then, the siRNA-SQ NPs were prepared by inverse nanoprecipitation : siRNA-SQ bioconjugates in water were added drop wisely to a stirred organic phase (acetone) with a volume/volume ratio of 1 [organic phase, acetone]: 2 [aqueous phase, siRNA-SQ bioconjugate]. Then acetone was evaporated using nitrogen (N2) flux to obtain an aqueous suspension of pure siRNA-SQ NPs. 


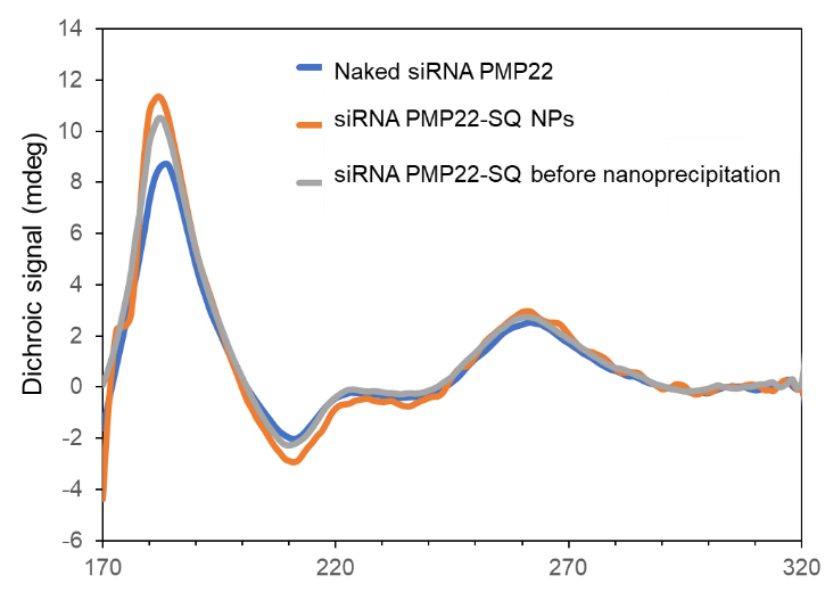

A

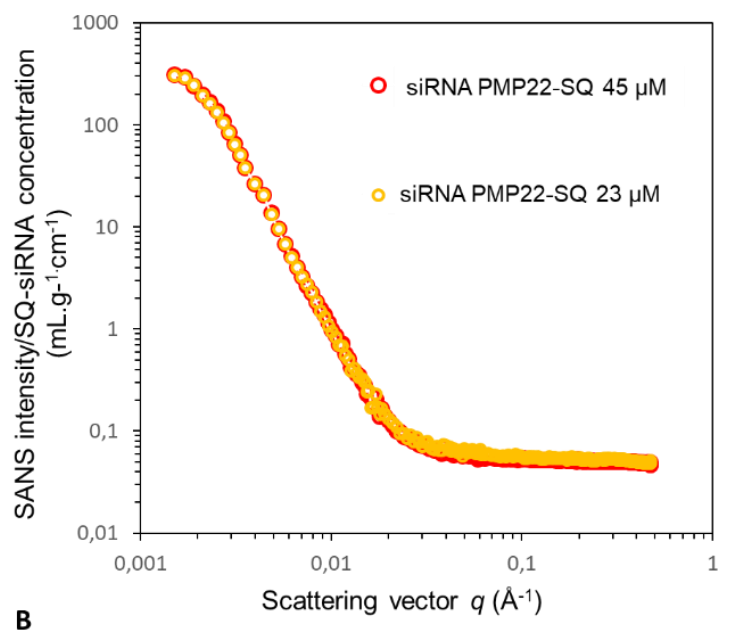

1007

Figure S2: Circular dichroism spectrum of siRNA. A) Naked siRNA (blue curve); siRNA-SQ NPs (orange) siRNA-SQ bioconjugates before nanoprecipitation (grey). Intensity difference (y-axis) is due to the use of different sample concentrations. B) Small-angle neutron scattering patterns of two dilutions of siRNA-SQ suspensions. Red circles: $45 \mu \mathrm{M}$; yellow circles: 23 $\mu \mathrm{M}$. The small-angle region corresponds to values of $\mathrm{q}<0.002 \AA^{-1}$, mean angles 0.002 to $0.04 \AA^{-1}$ and the large-angle $\mathrm{q}>0.04 \AA^{-1}$. 


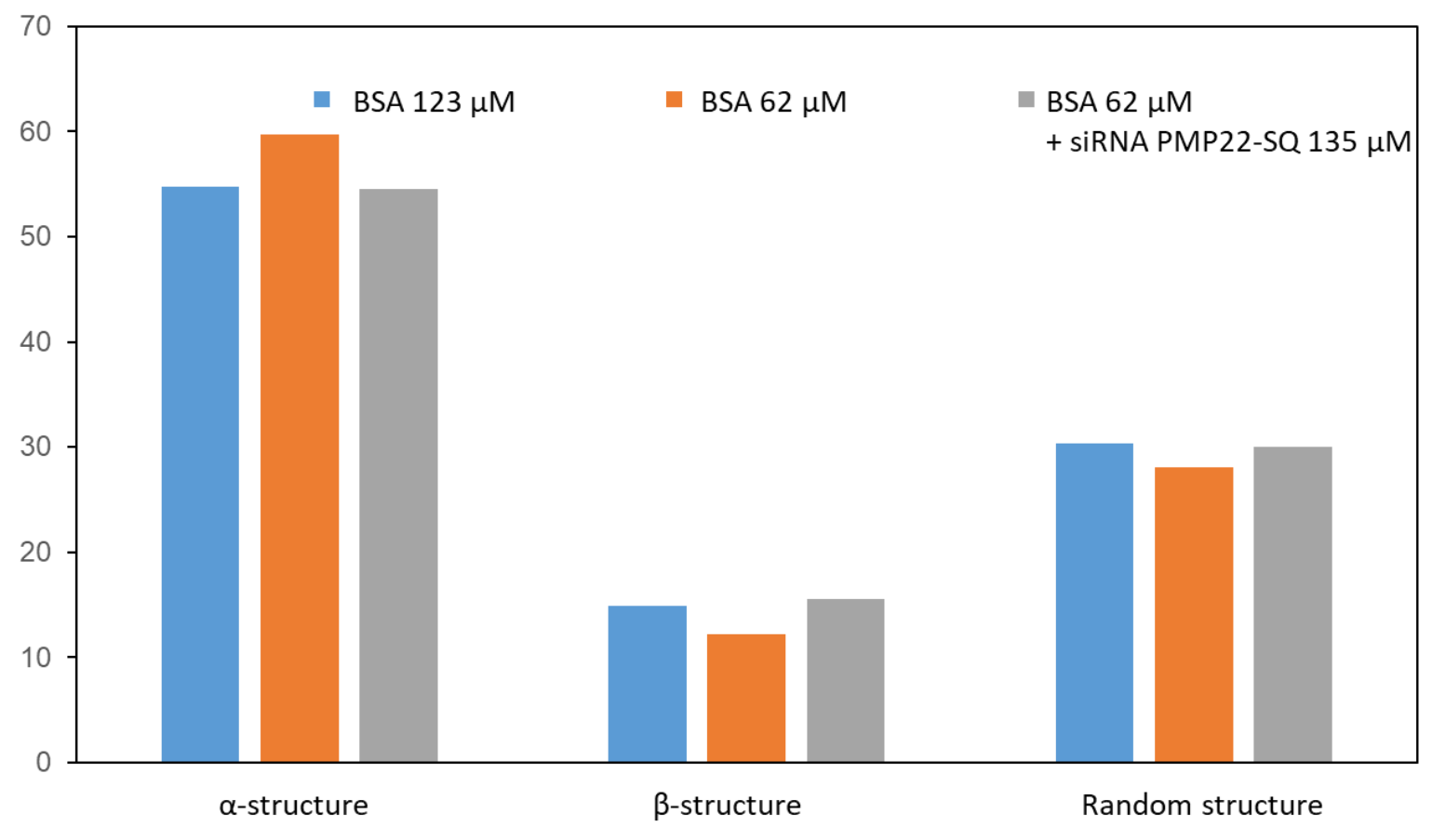

1016

Figure S3: Quantification of the secondary structure of BSA. After circular dichroism 1018 analysis, quantifications of BSA were performed by BestSel program 1019 (http://bestsel.elte.hu/index.php). The histogram represents the comparison of two BSA 1020 concentrations ( $123 \mu \mathrm{M}$ in blue and $62 \mu \mathrm{M}$ in orange) alone or in presence of siRNA-SQ NPs 1021 (in grey) with BSA at $62 \mu \mathrm{M}$. No difference was observed for the different conditions. 
Table S1: Table comparing siRNA size estimation

\begin{tabular}{|l|l|}
\hline "Source" & Dimensions $(\mathbf{n m})$ \\
\hline 22 base pairs $x$ 3.5 $\AA$ & $\mathrm{L}=7 \mathrm{~nm}$ \\
\hline & Cylindrical form factor \\
SANS pattern Ristori et al. & $\begin{array}{l}\mathrm{L}=5.2 \mathrm{~nm} \\
\mathrm{R}=1.1 \mathrm{~nm}\end{array}$ \\
\hline & $\begin{array}{l}\text { Cylindrical form factor } \\
\text { SAXS pattern of siRNA strands }\end{array}$ \\
$\mathrm{L}=7 \mathrm{~nm}$ \\
$\mathrm{R}=1.1 \mathrm{~nm}$ \\
\hline SAXS pattern of siRNA-SQ & Cylindrical form factor \\
& $\mathrm{L}=4 \mathrm{~nm}$ \\
$\mathrm{R}=1.1 \mathrm{~nm}$
\end{tabular}
oscillation in the same $q$-range in accordance with the SANS analysis of similar siRNA strands reported by Ristori et al. (Ristori, Ciani et al. 2012). 
1031

1032

1033

1034

1035

1036

1037

1038

$$
\frac{F_{0}}{F}=1+\mathrm{K}_{\mathrm{sv}}[\mathrm{siRNA} \text { PMP22-SQ }]=1+\mathrm{K}_{\mathrm{q}} \tau_{0}[\operatorname{siRNA} \text { PMP22-SQ }]
$$

Equation A. Stern-Volmer equation: $F_{0}$ and $F$ are fluorescence intensities of BSA in absence and presence of quencher (siRNA-SQ NPs), [siRNA PMP22-SQ] the molar concentration of siRNA PMP22-SQ (in $\mathrm{M}$ ), $\mathrm{K}_{\mathrm{sv}}\left(\mathrm{M}^{-1}\right)$ the Stern-Volmer constant which depends on $\mathrm{K}_{\mathrm{q}}\left(\mathrm{M}^{-1} \cdot \mathrm{s}^{-1}\right)$ and $\tau_{0}(\mathrm{~s})$ the average lifetime of tryptophan fluorescence in the absence of quencher, equals $10^{-8} \mathrm{~s}$. 
1040 Interaction between the siRNA-SQ nanoparticles (NPs) and BSA can be probed in the 1041 following formula:

1042 (B1) siRNAPMP22-SQ NPs $+\mathrm{n}(\mathrm{BSA}) \leftrightarrow[$ siRNA PMP22-SQ NPs-(BSA) $]$

1043 According to this equation we can determine the apparent dissociation constant $\left(\mathrm{K}_{\mathrm{obs}}\right)$ at $\mathrm{pH} 7$ 1044 and at $25^{\circ} \mathrm{C}$.

1045

1046

$$
\mathrm{K}_{\mathrm{obs}}=\frac{[\text { siRNA PMP22-SQ][BSA }]}{[\text { siRNA PMP22-SQ-(BSA)] }}
$$

1047

1048

Based on equation (1), the intrinsic fluorescence intensity of the mixture is expressed as a function of the concentrations of all species in solution, weighted by experimental factors $\mathrm{f}_{1}$, $\mathrm{f}_{2}$ and $\mathrm{f}_{3}$ for [siRNA PMP22-SQ], [BSA] and |siRNA PMP22-SQ-BSA] respectively:

$$
\mathrm{F}=\mathrm{f}_{1}[\operatorname{siRNA} P M P 22-\mathrm{SQ}]+\mathrm{f}_{2}[\mathrm{BSA}]+\mathrm{f}_{3}\left[\operatorname{siRNA} P M P 22-\mathrm{SQ}-(\mathrm{BSA})_{\mathrm{n}}\right]
$$

1053

In absence of BSA, the initial fluorescence intensity is given by: $\quad \mathrm{F}_{0}=\mathrm{f}_{1}[\operatorname{siRNA} P M P 22-\mathrm{SQ}]_{\text {initial }}$ 1055

The experimental conditions are as follow: 1) ratio [BSA]: [siRNA-SQ] < 1; 2) the fluorescence intensity emitted by the siRNA PMP22-SQ NPs + BSA mixture should be completely adsorbed BSA, therefore 3$)$ the intensity emitted from free BSA $\left.\left(f_{2} \mid B S A\right]\right)$ can be neglected.

1060 The experimental factor $\mathrm{f}_{3}$ is calculated based on raw data. From the variation in fluorescence 1061 emission $\Delta \mathrm{F}=\mathrm{F}-\mathrm{F}_{0}$ of equation (3) the $\mathrm{K}_{\mathrm{obs}}$ (2) can be calculated from the linear leastsquares regression:

$$
\frac{\mathrm{f}_{3}-\mathrm{f}_{1}}{\Delta \mathrm{F}}=\frac{1}{[\text { siRNA PMP22-SQ }]_{\text {initial }}}+\frac{\mathrm{K}_{\text {obs }}}{[\text { siRNA PMP22-SQ }]_{\text {initial }}\left([\text { BSA }]-\frac{\Delta \mathrm{F}}{\mathrm{f}_{3}-\mathrm{f}_{1}}\right)}
$$


As serum components will interact with the squalene moiety which surround the nanoparticle, we postulated that the mass of one nanoparticle is based on its density $\left(\rho_{\mathrm{SQ}}=\right.$ $858 \mathrm{~kg} / \mathrm{m}^{3}$ ) and the nanoparticle volume:

1073

$$
\mathrm{m}_{\mathrm{NP}}=\rho_{\mathrm{SQ}} \mathrm{V}_{\mathrm{NP}}
$$

1074

Number of NPs can be calculated as follow:

1076

1077

$$
\mathrm{N}_{\mathrm{NP}}=\frac{\mathrm{C}_{\mathrm{m}}(\mathrm{siRNA} \text { PMP22-SQ })}{\mathrm{m}_{\mathrm{NP}}}
$$

1078

2) Determination of the number of proteins

Based on electrophoresis experimental conditions and molecular weight of the BSA, we

$$
\text { Number } \mathrm{BSA} / \mathrm{NP}=\frac{\mathrm{N}_{\mathrm{BSA}}}{\mathrm{m}_{\mathrm{NP}}}
$$

Where $[\mathrm{BSA}]$ is the molar concentration of BSA, $\mathrm{MM}_{\mathrm{BSA}}$ the BSA molecular weight $(65,000$ $\mathrm{g} / \mathrm{mol}$ ) and $\mathrm{V}$ the volume used for incubation before electrophoresis.

Number of BSA proteins can be determined as follow:

$$
\mathrm{N}_{\mathrm{BSA}}=\frac{\mathrm{m}_{\text {total BSA }}}{\mathrm{MM}_{\mathrm{BSA}}} \mathrm{N}_{\mathrm{A}}
$$

Where $\mathrm{N}$ is the BSA protein number and $\mathrm{N}_{\mathrm{A}}$ the Avogadro number (number of elementary entities contained in a mole of matter, equal to $6.023 \times 10^{23}$ ).

\section{3) Determination of BSA proteins per nanoparticle}

Based on (2) and (4), we can calculate the number of BSA interacting with one nanoparticle: 